\title{
Final-state interactions and superscaling in the semi-relativistic approach to quasielastic electron and neutrino scattering
}

\author{
J. E. Amaro, ${ }^{1}$ M. B. Barbaro, ${ }^{2}$ J. A. Caballero, ${ }^{3}$ T. W. Donnelly, ${ }^{4}$ and J. M. Udias ${ }^{5}$ \\ ${ }^{1}$ Departamento de Física Atómica, Molecular y Nuclear, Universidad de Granada, E-18071 Granada, Spain \\ ${ }^{2}$ Dipartimento di Fisica Teorica, Università di Torino and INFN, Sezione di Torino Via P. Giuria 1, I-10125 Torino, Italy \\ ${ }^{3}$ Departamento de Física Atómica, Molecular y Nuclear, Universidad de Sevilla, Apdo. 1065, E-41080 Sevilla, Spain \\ ${ }^{4}$ Center for Theoretical Physics, Laboratory for Nuclear Science and Department of Physics, Massachusetts Institute of Technology, \\ Cambridge, Massachusetts 02139, USA \\ ${ }^{5}$ Departamento de Física Atómica, Molecular y Nuclear, Universidad Complutense de Madrid, E-28040 Madrid, Spain
}

(Received 13 December 2006; published 22 March 2007)

\begin{abstract}
The semi-relativistic approach to electron and neutrino quasielastic scattering from nuclei is extended to include final-state interactions. Starting with the usual nonrelativistic continuum shell model, the problem is relativized by using the semi-relativistic expansion of the current in powers of the initial nucleon momentum and relativistic kinematics. Two different approaches are considered for the final-state interactions: the Smith-Wambach $2 \mathrm{p}-2 \mathrm{~h}$ damping model and the Dirac-equation-based potential extracted from a relativistic mean-field plus the Darwin factor. Using the latter, the scaling properties of $\left(e, e^{\prime}\right)$ and $\left(v_{\mu}, \mu^{-}\right)$cross sections for intermediate momentum transfers are investigated.
\end{abstract}

DOI: 10.1103/PhysRevC.75.034613

PACS number(s): 24.10.Jv, 21.60.Cs, 25.30.Fj, 25.30.Pt

\section{INTRODUCTION}

In recent years, the so-called semi-relativistic (SR) approach has been explored as a convenient and easily implementable way to "relativize" existing Schrödinger-based models of quasielastic (QE) electron scattering from nuclei [1-6]. This approach (which differs from other approaches also called "semi-relativistic" in the literature $[7,8]$ ) is based on the SR expansion of the on-shell electromagnetic current in powers of $p / m_{N}$ only, i.e., the momentum of the initial nucleon divided by the nucleon mass, leaving untouched the dependence on the momentum transfer $q$ and energy transfer $\omega$. Implementing this current expansion and employing relativistic kinematics, the resulting SR models can be applied at large values of the momentum transfer, of order of a $\mathrm{GeV}$ or more, where the traditional relativistic corrections [9] based on expansions of the current in powers of $1 / m_{N}$ are bound to fail, whatever the order of the expansion. The existence in the literature [10] of expansions up to fourth order, at least in a Foldy-Wouthuysen transformation where they are seen to be quite complicated, raises simplicity as another advantage of the SR approach. In fact, by carefully grouping the expansion terms, one can write them as $(q, \omega)$-dependent factors times the traditional [11,12] leading-order charge, convection, and spin-magnetization current operators [1]. Moreover, the SR first-order $O\left(p / m_{N}\right)$ correction to the charge operator [13] is proportional to the spin-orbit charge operator $i \vec{q} \cdot(\vec{\sigma} \times \vec{p})$, usually obtained in the second-order $1 / m_{N}^{2}$ correction in traditional nonrelativistic expansions [14].

Extensive tests of the SR expansion have been performed within the context of the Fermi gas. In particular, comparisons with the fully relativistic Fermi gas (RFG), where the exact result is well known, have shown the reliability of the expansion [1]. Similar SR expansions have also been performed for meson-exchange currents [15] and nucleon- $\Delta$ currents [16] and, more recently, for the charge-changing $(\mathrm{CC})$ weak current driving $\mathrm{QE}\left(v_{l}, l^{-}\right)$reactions [17].

Part of the interest in reactions involving neutrinos instead of electrons lies in their implications for ongoing and planned neutrino oscillation experiments [18-26]. Since these naturally involve heavy nuclei as targets, reliable nuclear models of the reaction $A\left(v_{l}, l^{-}\right)$play an essential role in the interpretation of the data. Because of the close relationships that exist among all semi-leptonic electroweak processes, accurate descriptions of $A\left(e, e^{\prime}\right)$ data appear as a requirement that sets strong restrictions on nuclear modeling of neutrino reactions. Based on what is known from previous approaches to this problem and on the results presented in this paper, two ingredients arise as being essential if the nuclear reaction modeling is to be successful in describing the electroweak cross sections in the kinematic regime of interest, namely, relativity and finalstate interactions (FSI).

First is relativity, since one is dealing with momentum transfers in the intermediate-to-high energy regime, typically of the order of $1 \mathrm{GeV}$ or higher, for which traditional nonrelativistic expansions in powers of $1 / m_{N}$ are not applicable. Different fully relativistic models (based on Dirac equations and/or relativistic many-body theories) have been developed in recent years aiming to describe electron and neutrino scattering [27-41]. While relativistic approaches based on the Dirac equation appear as the most direct way to deal with the problem, the importance of the different relativistic ingredients is more easily explored by detailed comparison with the extensively employed nonrelativistic approaches, and particularly with the SR one that is the focus of this work. This comparison will also help in identifying which relativistic ingredients (current operators, initial- or finalstate wave functions) are the main ones responsible for the 
difference between relativistic and nonrelativistic results. Upon incorporating these ingredients in SR approaches, one can extend their applicability to regions well into the relativistic domain and thereby hope to produce reliable results at high energies.

Second, the model of FSI must account for many-body effects that are known to be essential in describing reasonably well $\left(e, e^{\prime}\right)$ cross sections within the context of nonrelativistic approaches [42-48]. For instance, medium modifications of the one-particle one-hole $(1 \mathrm{p}-1 \mathrm{~h})$ final-state self-energy via two-particle two-hole $(2 \mathrm{p}-2 \mathrm{~h})$ intermediate states in the continuum appear to be important in nonrelativistic manybody treatments of a variety of processes involving low-tointermediate momentum transfers [49-51]. The main effect is a significant shift of strength to high energies in the $\mathrm{QE}$ cross section. On the other hand, relativistic impulse approximation modeling of inclusive reactions [41,52] shows an important effect of the FSI where strength is shifted to the high momentum region.

The importance of FSI for intermediate-to-high momentum transfers has also been investigated in connection with the scaling properties of the QE cross section. The recent analysis of $\left(e, e^{\prime}\right)$ world data in the QE region [53-55] has permitted the extraction of a universal scaling function $f_{L}\left(\psi^{\prime}\right)$ from the longitudinal response function data [here $\psi^{\prime}$ is the dimensionless scaling variable defined in Eq. (29) below]. The experimental scaling function presents an asymmetric shape, in contrast to the symmetric behavior predicted by most independentparticle models, which essentially give results that are similar to the RFG, with the exception of the tails observed for $\psi^{\prime}<-1$ and $\psi^{\prime}>1$, where the RFG result is zero by construction. The behavior exhibited by the experimental scaling function reflects that of the longitudinal response function including the FSI for intermediate values of the momentum transfer [45], i.e., the single-particle strength at the QE peak is reduced and a large tail is observed for high values of the energy transfer $\omega$. Note that the difference with respect to previous approaches is that the experimental data now correspond to relatively high values of the momentum transfer, mainly in the range from $q=$ $500 \mathrm{MeV} / c$ to $1 \mathrm{GeV} / c$, for which scaling occurs, and where most of the existing nonrelativistic models of FSI are no longer applicable.

Recent work [41,52] has reported good agreement between the calculated scaling function and the data in a fully relativistic approach based on the relativistic mean-field (RMF) model, within the impulse approximation (IA). The bound and outgoing nucleon states are described by the same self-consistent Dirac-Hartree potential. Here, the use of the same relativistic potential in the initial and final states appears to be essential, since, as shown in Ref. [52], calculations using the real parts of complex relativistic optical potentials do not produce the asymmetric behavior seen in the data. Apparently the main factors responsible for the asymmetry are not only relativistic kinematics, but also the particular dynamics contained in the Dirac equation when strong scalar and vector potentials are used for the continuum nucleon states (i.e., for the FSI). In fact, within the IA, the significant shift of strength to positive $\psi^{\prime}$ values only occurs under the presence of these strong potentials [41].
In this paper, we investigate whether it is possible to describe the asymmetric behavior of the QE scaling function within the semi-relativistic approach for intermediate-to-high momentum transfers. As FSI are essential in order to perform meaningful comparisons with QE scaling data, we explore various ways of including these ingredients in the SR model at relativistic energies. We consider two different models of FSI: the Smith-Wambach 2p-2h damping (SWD) model and the Dirac-equation-based (DEB) potential plus the Darwin factor $(\mathrm{DEB}+\mathrm{D})[30,56,57]$.

The SWD model is an extrapolation to relativistic kinematics of the nuclear reinteraction model introduced in Refs. [43,49], which gives one a straightforward way to incorporate the effects of $2 \mathrm{p}-2 \mathrm{~h}$ excitations in the nuclear response function within a nonrelativistic context. Speculations on how to extend this model to $\mathrm{QE}$ neutrino scattering for relativistic energies have been presented in Refs. [58,59], within the context of the RFG.

The DEB+D method attempts to translate the success of the RMF model in describing the phenomenological scaling function from $\mathrm{QE}\left(e, e^{\prime}\right)$ data $[41,52]$ to the SR approach. We perform calculations in a SR continuum shell model where the final wave functions are obtained by solving the Schrödinger equation with the DEB potential and multiplying by the Darwin factor. The DEB potential is obtained by a reduction of the Dirac equation to a Schrödinger equation with a local potential, which implies that one must multiply the wave function by a nonlocality Darwin factor $[30,56,57,60]$. This essentially amounts to using upper components from the solutions of the Dirac equation in computing the final nucleon wave functions.

Both the DEB potential and Darwin factor are functions of the local (energy independent) vector $(V)$ and scalar $(S)$ components of the relativistic Hartree potential. The nonrelativistic reduction implied in the derivation of the DEB potential and the Darwin term introduces a linear dependency on the energy of the particle. The results obtained in the SR model describing FSI in this way compare well with those obtained using the fully relativistic RMF model, and thus also reproduce the successful comparison with the experimental scaling function data. This allows us to conclude that it is the treatment of FSI in the RMF that gives rise to the large asymmetric tail in the superscaling function.

In this paper, we also present an application of the SR-DEB+D approach to the superscaling analysis (SuSA) of the neutrino $\mathrm{CC}$ QE cross section. This method has been proposed [61] as an efficient way of predicting neutrino cross sections from the $\left(e, e^{\prime}\right)$ data, by exploiting the scaling properties of the latter. The method is based on the hypothesis that the neutrino cross sections universally scale in the same way as do the electron scattering cross sections for intermediateto-high momentum transfers, as seen for several types of models $[17,41,52]$. An application of the SuSA approach to compute integrated neutrino cross sections has been reported recently [62]. In this work, we investigate the validity of the superscaling approach within the SR-DEB +D model. Given the success of our model in describing the experimental scaling data, this check will help to further lay the foundations of the SuSA approach introduced originally in Ref. [61]. 
The structure of the work is the following. In Sec. II we briefly outline the SR model and the different treatments of the FSI. We present results in Sec. III and our conclusions in Sec. IV.

\section{SEMI-RELATIVISTIC (SR) MODEL}

In this section, we summarize the basic formalism for electron and neutrino reactions within the SR approach, including the different treatments of the FSI. We refer the reader to Refs. [1,17] for specific details.

\section{A. Electron and neutrino cross sections}

We focus specifically on the reactions $\left(e, e^{\prime}\right)$ and $\left(v_{l}, l^{-}\right)$ induced by electrons and neutrinos, respectively, where $l^{-}$is a lepton with mass $m_{l}$ (typically a muon). The four-momentum of the incident lepton is $k^{\mu}=(\epsilon, \mathbf{k})$, while $k^{\prime \mu}=\left(\epsilon^{\prime}, \mathbf{k}^{\prime}\right)$ is the four-momentum of the final lepton. The four-momentum transfer is denoted $Q^{\mu}=k^{\mu}-k^{\prime \mu}=(\omega, \mathbf{q})$. The results below are referred to a coordinate system where the $z$ axis points along $\mathbf{q}$ and the $x$ axis along $\mathbf{k}-(\mathbf{k} \cdot \mathbf{q}) \mathbf{q} / q^{2}$.

The inclusive cross section for these reactions can be written in general as

$$
\frac{d \sigma}{d \Omega^{\prime} d \epsilon^{\prime}}=\sigma_{0} \mathcal{F}^{2},
$$

where $\sigma_{0}$ is the usual Mott cross sections for $\left(e, e^{\prime}\right)$ reactions [4], while it becomes an analogous factor in the case of $\left(v_{l}, l^{-}\right)$ reactions (see Refs. [17,61] for explicit expressions). The relevant observable is the nuclear structure function $\mathcal{F}^{2}$ which can be written as

$$
\mathcal{F}^{2}=v_{L} R_{L}+v_{T} R_{T}
$$

for $\left(e, e^{\prime}\right)$ and

$$
\begin{aligned}
\mathcal{F}^{2}= & \hat{V}_{\mathrm{CC}} R_{\mathrm{CC}}+2 \hat{V}_{\mathrm{CL}} R_{\mathrm{CL}} \\
& +\hat{V}_{\mathrm{LL}} R_{\mathrm{LL}}+\hat{V}_{T} R_{T}+2 \hat{V}_{T^{\prime}} R_{T^{\prime}}
\end{aligned}
$$

for $\left(v_{l}, l^{-}\right)$reactions. The lepton kinematic factors $v_{K}$ and $\hat{V}_{K}$ are defined in Refs. [4] and [61], respectively. Note that in the limit of lepton mass $m_{l}=0$, one has the identities $v_{L}=\hat{V}_{\mathrm{CC}}$ and $v_{T}=\hat{V}_{T}$. Finally, the nuclear response functions are given by

$$
\begin{aligned}
R_{L} & =R_{\mathrm{CC}}=W^{00}, \\
R_{\mathrm{CL}} & =-\frac{1}{2}\left(W^{03}+W^{30}\right), \\
R_{\mathrm{LL}} & =W^{33}, \\
R_{T} & =W^{11}+W^{22}, \\
R_{T^{\prime}} & =-\frac{1}{2}\left(W^{12}-W^{21}\right),
\end{aligned}
$$

where the inclusive hadronic tensor is

$$
\begin{aligned}
W^{\mu \nu}= & \sum_{f i}^{-} \delta\left(E_{f}-E_{i}-\omega\right)\left\langle f\left|J^{\mu}(Q)\right| i\right\rangle^{*} \\
& \times\left\langle f\left|J^{\nu}(Q)\right| i\right\rangle .
\end{aligned}
$$

Here $J^{\mu}(Q)$ is the nuclear current operator relevant for the reaction, i.e., the electromagnetic current in the case of $\left(e, e^{\prime}\right)$ or the CC weak current in the case of $\left(v_{l}, l^{-}\right)$, as specified below. Note that although in Eq. (4) $R_{L}$ and $R_{\mathrm{CC}}$ are formally equal, in practice they are not the same quantity, since different current operators and nuclear matrix elements are involved in their definitions, $R_{L}$ referring to electron scattering and $R_{\mathrm{CC}}$ to neutrino reactions.

The current operators used in this work are SR expansions of the fully relativistic ones in powers of $\eta=\mathbf{p} / m_{N}$, where $\mathbf{p}$ is the momentum of the initial (bound) nucleon, which is typically small $(\eta<1 / 4)$, and therefore an expansion at most to first order $O(\eta)$ should be adequate for the inclusive reactions considered here in the region of the QE peak. The expansion was performed in Refs. [1,17] (see Ref. [4] for a review on the general SR expansion procedure). Here we give just the final expressions used in our calculations. The CC weak current is written in momentum space as $J^{\mu}=J_{V}^{\mu}-J_{A}^{\mu}$, in terms of the vector and axial current terms. For the vector current, one has

$$
\begin{aligned}
J_{V}^{0} & =\xi_{0}+i \xi_{0}^{\prime}(\boldsymbol{\kappa} \times \boldsymbol{\eta}) \cdot \boldsymbol{\sigma}, \\
\mathbf{J}_{V}^{\perp} & =\xi_{1} \boldsymbol{\eta}^{\perp}+i \xi_{1}^{\prime} \boldsymbol{\sigma} \times \boldsymbol{\kappa},
\end{aligned}
$$

and for the axial current,

$$
\begin{aligned}
& J_{A}^{0}=\zeta_{0}^{\prime} \kappa \cdot \sigma+\zeta_{0}^{\prime \prime} \eta^{\perp} \cdot \sigma, \\
& J_{A}^{z}=\zeta_{3}^{\prime} \kappa \cdot \sigma+\zeta_{3}^{\prime \prime} \eta^{\perp} \cdot \sigma, \\
& \mathbf{J}_{A}^{\perp}=\zeta_{1}^{\prime} \sigma^{\perp} .
\end{aligned}
$$

In Eqs. (10)-(14), we have introduced the dimensionless momentum transfer vector $\boldsymbol{\kappa}=\mathbf{q} / 2 m_{N}$, while we use the notation $\mathbf{J}^{\perp}$ to denote the component of the vector $\mathbf{J}$ perpendicular to the momentum transfer $\mathbf{q}$.

Finally, the nucleon form factors and relativistic correction factors are included in the coefficients $\xi_{i}, \xi_{i}^{\prime}, \zeta_{i}^{\prime}$, and $\zeta_{i}^{\prime \prime}$ (the corresponding relativistic versions of these quantities for the electroweak neutral current were introduced in the Appendix of Ref. [1]), defined by

$$
\begin{aligned}
& \xi_{0}=\frac{\kappa}{\sqrt{\tau}} 2 G_{E}^{V}, \xi_{0}^{\prime}=\frac{2 G_{M}^{V}-G_{E}^{V}}{\sqrt{1+\tau}} \\
& \xi_{1}^{\prime}=2 G_{M}^{V} \frac{\sqrt{\tau}}{\kappa}, \quad \xi_{1}=2 G_{E}^{V} \frac{\sqrt{\tau}}{\kappa} \\
& \zeta_{0}^{\prime}=\frac{1}{\sqrt{\tau}} \frac{\lambda}{\kappa} G_{A}^{\prime}, \quad \zeta_{0}^{\prime \prime}=\frac{\kappa}{\sqrt{\tau}}\left[G_{A}-\frac{\lambda^{2}}{\kappa^{2}+\kappa \sqrt{\tau(\tau+1)}} G_{A}^{\prime}\right]
\end{aligned}
$$

$$
\zeta_{3}^{\prime}=\frac{1}{\sqrt{\tau}} G_{A}^{\prime}, \quad \zeta_{3}^{\prime \prime}=\frac{\lambda}{\sqrt{\tau}}\left[G_{A}-\frac{\kappa}{\kappa+\sqrt{\tau(\tau+1)}} G_{A}^{\prime}\right],
$$

$$
\zeta_{1}^{\prime}=\sqrt{1+\tau} G_{A}
$$

Here use has been made of the dimensionless variables $\kappa=$ $q / 2 m_{N}, \lambda=\omega / 2 m_{N}$, and $\tau=\kappa^{2}-\lambda^{2}$. The nucleon form factors appearing in Eqs. (15)-(19) are the isovector magnetic $G_{M}^{V}=G_{M}^{p}-G_{M}^{n}$, isovector electric $G_{E}^{V}=G_{E}^{p}-G_{E}^{n}$, and the axial form factor $G_{A}$. In $\zeta_{i}^{\prime}$ and $\zeta_{i}^{\prime \prime}$ we have introduced the 
following combination of axial-vector and pseudoscalar form factors:

$$
G_{A}^{\prime}=G_{A}-\tau G_{P},
$$

where $G_{P}$ is the pseudoscalar nucleon form factor.

The expression for the SR expansion of the electromagnetic current is very similar to that for the vector current in Eqs. (10) and (11). The only difference is that the proper proton or neutron form factors, instead of the isovector ones, should be inserted in the corresponding $\xi_{i}$ and $\xi_{i}^{\prime}$ coefficients.

\section{B. Nuclear and FSI models}

In this work, we describe the nuclear structure with an uncorrelated shell model. The many-body initial nuclear state $|i\rangle$ is a Slater determinant. It is constructed with a set of singleparticle solutions of a nonrelativistic mean-field potential. The final states $|f\rangle=\left|\mathrm{ph}^{-1}\right\rangle$ are particle-hole excitations of the nuclear core. The holes $|h\rangle$, with nonrelativistic energies $\epsilon_{h}$, are solutions of the Schrödinger equation with a Woods-Saxon potential. The particles $|p\rangle$, with asymptotic kinetic energies $\epsilon_{p}=\epsilon_{h}+\omega$, are continuum solutions of the Schrödinger equation with positive energy. In the SR approach, the relativistic kinematics is implemented by the following substitution of the eigenvalue for positive energies:

$$
\epsilon_{p} \rightarrow \epsilon_{p}\left(1+\frac{\epsilon_{p}}{2 m_{N}}\right)
$$

For intermediate energies, $\epsilon_{h}$ is small compared with $\omega$ and the above substitution can be replaced to a good approximation by $\lambda \rightarrow \lambda(1+\lambda)$ with small error [63].

We begin at the basic starting point, denoted below as semirelativistic Woods-Saxon (SR-WS), where the continuum states are described by solutions of the Schrödinger equation with the same Woods-Saxon potential as the bound states. Building on this, two further different approaches to FSI have been considered.

In a first treatment, we include the FSI effects coming from $2 \mathrm{p}-2 \mathrm{~h}$ intermediate states by following the Smith-Wambach damping model (SWD). Within this model the total response function $R_{K}^{\mathrm{SWD}}(q, \omega)$ is computed from the bare SR-WS one, $R_{K}^{\mathrm{SR}-\mathrm{WS}}$, as a folding or convolution integral

$$
\begin{aligned}
R_{K}^{\mathrm{SWD}}(q, \omega)= & \int_{0}^{\infty} d E R_{K}^{\mathrm{SR}-\mathrm{WS}}(q, E)\left[\rho\left(\frac{m_{N}}{M^{*}(q)} E, \omega\right)\right. \\
& \left.+\rho\left(\frac{m_{N}}{M^{*}(q)} E,-\omega\right)\right],
\end{aligned}
$$

where $M^{*}(q)$ is the nucleon effective mass taken from Ref. [64] and, following Ref. [49], the function $\rho(E, \omega)$ is given by

$$
\begin{aligned}
\rho(E, \omega) & =\frac{1}{\pi} \frac{\Gamma(\omega) / 2}{[E-\omega-\Delta(\omega)]^{2}+[\Gamma(\omega) / 2]^{2}}, \\
\Sigma(\omega) & =\Delta(\omega)+i \frac{1}{2} \Gamma(\omega)
\end{aligned}
$$

being the complex self-energy of the final states. The imaginary self-energy is computed through the following average for the particles and holes:

$$
\Gamma(\omega)=\frac{1}{\omega} \int_{0}^{\omega} d \epsilon\left[\gamma_{p}(\epsilon)+\gamma_{h}(\epsilon-\omega)\right] .
$$

We use the parametrization of Ref. [65]

$$
\gamma_{p}(\epsilon)=\gamma_{h}(\epsilon)=\gamma(\epsilon)=2 \alpha\left[\frac{\epsilon^{2}}{\epsilon^{2}+\epsilon_{0}^{2}}\right]\left[\frac{\epsilon_{1}^{2}}{\epsilon^{2}+\epsilon_{1}^{2}}\right],
$$

with $\alpha=10.75 \mathrm{MeV}, \epsilon_{0}=18 \mathrm{MeV}$, and $\epsilon_{1}=110 \mathrm{MeV}$.

The second approach to FSI, and the main focus in this work, is the Dirac-equation-based plus Darwin term $(\mathrm{DEB}+\mathrm{D})$. The DEB potential $U_{\mathrm{DEB}}$ is obtained from the Dirac equation, written as a second-order equation for the upper component $\psi_{u p}(\mathbf{r})[30,56,57]$. This wave function is then written in the form

$$
\psi_{u p}(\mathbf{r})=K(r, E) \phi(\mathbf{r}),
$$

and the Darwin factor $K(r, E)$ is chosen in such a way that the function $\phi(r)$ satisfies a Schrödinger-like equation

$$
\left[-\frac{1}{2 m_{N}} \nabla^{2}+U_{\mathrm{DEB}}(r, E)\right] \phi(\mathbf{r})=\frac{E^{2}-m_{N}^{2}}{2 m_{N}} \phi(\mathbf{r}) .
$$

Here $E$ is the relativistic energy of the final nucleon. Note that both the DEB potential $U_{\mathrm{DEB}}(r, E)$ and the Darwin factor $K(r, E)$ show an explicit energy dependence coming from the nonrelativistic reduction of the Dirac equation. The complete expressions in terms of the vector and scalar parts of the relativistic potential are given, for instance, in Refs. [30,60]. In the results section, we show the energy dependence of these quantities in more detail.

\section{Superscaling approach}

In this paper, we focus on the properties of the scaling functions $f_{L}\left(\psi^{\prime}\right)$ and $f_{T}\left(\psi^{\prime}\right)$; these are defined as the electromagnetic responses $R_{L}(q, \omega)$ and $R_{T}(q, \omega)$ divided by the appropriate single-nucleon functions, $G_{K}(K=L, T)$, weighted by the nucleon number involved in the process (see Refs. [17,61] for details).

The scaling functions depend generally on $q$ and $\omega$ and are different for different nuclei. Scaling of first kind is said to hold when they are found to depend, not independently on $q$ and $\omega$, but only on a specific function of these two, namely, the scaling variable $\psi^{\prime}(q, \omega)$ defined as

$$
\psi^{\prime}=\xi_{F}^{-1 / 2} \frac{\lambda^{\prime}-\tau^{\prime}}{\left[\left(1+\lambda^{\prime}\right) \tau^{\prime}+\kappa \sqrt{\tau^{\prime}\left(1+\tau^{\prime}\right)}\right]^{1 / 2}},
$$

where $\lambda^{\prime}=\left(\omega-E_{s}\right) / 2 m_{N}, \kappa=q / 2 m_{N}, \tau^{\prime}=\kappa^{2}-\lambda^{2}$, and $\xi_{F}=\sqrt{1+\left(k_{F} / m_{N}\right)^{2}}-1$. Experimentally, the Fermi momentum $k_{F}$ and the energy shift $E_{s}$ are empirical parameters determined through fits to $\mathrm{QE}$ electron scattering data. The unshifted scaling variable $\psi$ is defined by the same formula, but with $E_{S}=0$.

If $f_{L}$ and $f_{T}$ are independent of $k_{F}$ as well, one says that second-kind scaling in fulfilled. When both kinds of scaling occur, the responses are said to "superscale." 
In the case of neutrino reactions, scaling functions can be obtained in a similar way, dividing the weak responses by the corresponding single-nucleon contribution, see Refs. $[17,41,61]$. The approach called SuSA assumes that the scaling functions entering both $\left(e, e^{\prime}\right)$ and $\left(\nu_{l}, l^{-}\right)$reactions are the same; in this case, one can reconstruct the weak cross sections from the electromagnetic ones by multiplying the scaling function by the weak single-nucleon contribution.

\section{RESULTS}

In this section, we present results for the nucleus ${ }^{12} \mathrm{C}$. The ground state is described here in the extreme shell model; namely, the $1 s_{1 / 2}$ and $1 p_{3 / 2}$ shells are fully occupied for protons and neutrons. The Woods-Saxon potential parameters used to describe the (nonrelativistic) bound energies and wave functions are given in Ref. [17]. As stated in the previous section, for the final states in the SR model, we use different forms of the potential depending on the description of the FSI. In the SR-WS, the same Woods-Saxon potential is used for initial and final states. In the SR-DEB, the DEB potential and the Darwin term are used for the final states. A general multipole expansion of the current operators is performed to compute the nuclear response functions and scaling functions. We refer the reader to Refs. [1,17] for details on this aspect of the calculation.

We first investigate the properties of the electromagnetic $\left(e, e^{\prime}\right) L$ and $T$ scaling functions, leaving the discussion of the neutrino CC cross section for the end of this section. In particular, we are interested in the study of the properties of scaling of the first kind. To this end, we compute the function $f_{K}\left(\psi^{\prime}\right)(K=L, T)$ for fixed values of the momentum transfer $q$. One has scaling of the first kind when no dependence on $q$ is observed.

\section{A. Relativistic effects}

We start with a brief discussion of the size of the relativistic effects embodied in the SR model. In Fig. 1, we show the longitudinal and transverse scaling functions of ${ }^{12} \mathrm{C}$ computed in the shell model for three values of the momentum transfer, $q=0.5,0.7$, and $1 \mathrm{GeV} / c$. We show results for three models of the reaction, all of them including the same Woods-Saxon potential in initial and final states. The dashed lines correspond to a traditional nonrelativistic approach using nonrelativistic currents and nonrelativistic kinematics. The dotted lines have been obtained using relativistic kinematics, but still the nonrelativistic current operators. Finally, the solid lines correspond to the SR approach with relativistic corrections in the current as well, i.e., to the SR-WS model.

As one can see, the width of the scaling functions is significantly reduced in the calculations that include relativistic kinematics, while the inclusion of relativistic effects in the current operators leads to an increase of $f_{L}$ and a reduction of $f_{T}$. The importance of relativistic effects clearly increases with the momentum transfer. In the figure, we also plot the experimental data for the averaged $f_{L}\left(\psi^{\prime}\right)$, taken from Ref. [55]. It is important to point out that data refer only to the analysis of the longitudinal scaling function, i.e., $f_{L}$; however,
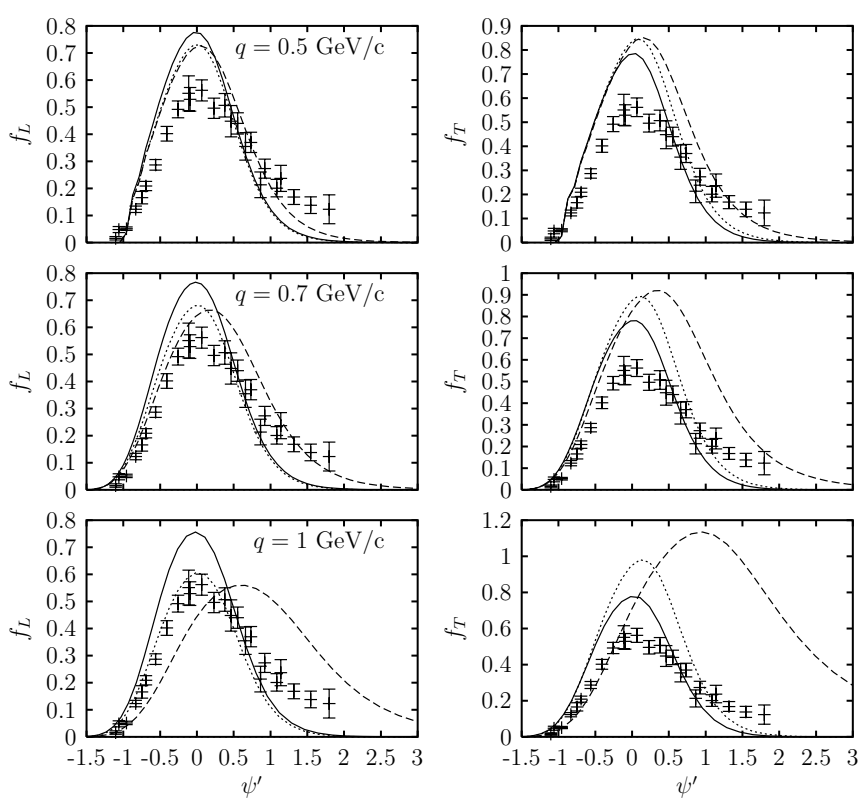

FIG. 1. Scaling functions of ${ }^{12} \mathrm{C}$ in the continuum shell model with a Woods-Saxon potential. Solid lines: SR model. Dashed: traditional nonrelativistic results. Dotted: nonrelativistic current operators using relativistic kinematics. Experimental data are from Ref. [55].

we have chosen to present such data also in comparison with theoretical results for $f_{T}$. This makes the comparison between $f_{L}$ and $f_{T}$ more straightforward and, in fact, it allows us to check the degree to which scaling of the zeroth kind, i.e., $f_{L}=f_{T}$, is fulfilled for the different models presented in this work.

Incidentally, note that the nonrelativistic current with relativistic kinematics (dotted lines) gives the best description of experimental data for $q=1 \mathrm{GeV} / c$, where a nonrelativistic treatment of the current operator should hardly be adequate. This fact is a consequence of the relativistic factor $\kappa / \sqrt{\tau}$ in the coefficient $\xi_{0}$ in Eq. (15) which produces an increase of $f_{L}$.

Although from the full set of results seen in Fig. 1 it clearly appears that none of the three approaches provides a satisfactory description of the experimental data, one can still study the scaling properties of these models. One knows from previous superscaling studies that scaling of the first kind is approximately valid for the experimental longitudinal scaling function, $f_{L}$. This places important restrictions on theoretical models, namely, that they should be (reasonably) compatible with scaling of the first kind. Otherwise they would be unable to describe the $\left(e, e^{\prime}\right)$ cross section over a wide range of momenta.

On the other hand, we recall that the scaling variable is obtained from a consideration of relativistic kinematics, and the scaling functions are obtained dividing by a single-nucleon cross section that is derived from a fully relativistic form. It is only when both these ingredients are included that the experimental data show scaling. It is hardly surprising that approximations to quasielastic scattering that do not contain the appropriate relativistic content fail to produce the appropriate scaling. 

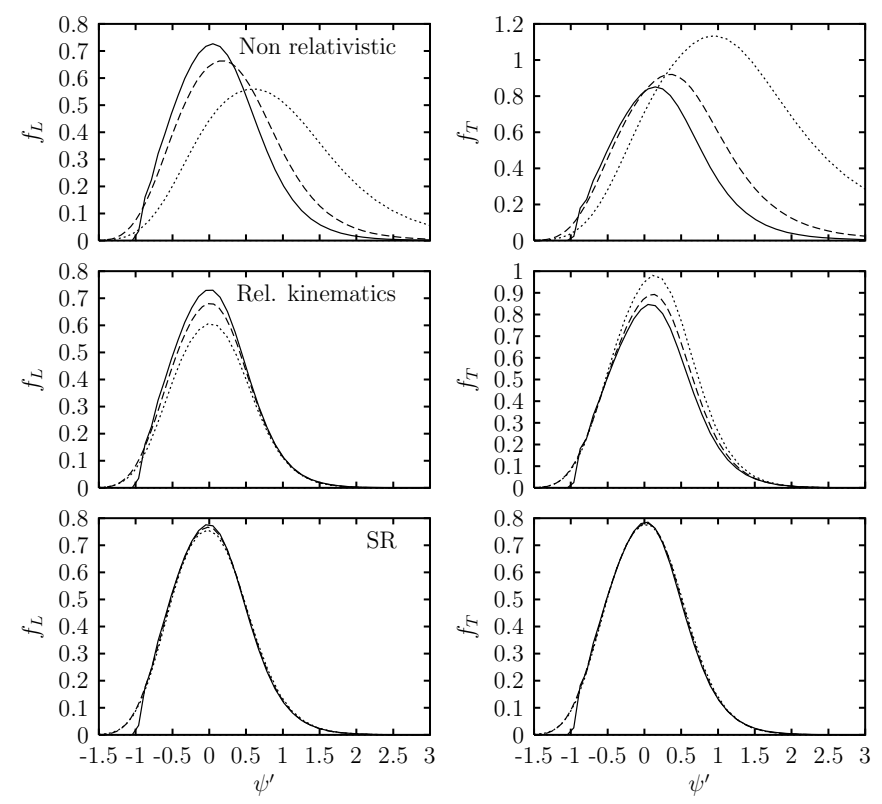

FIG. 2. ${ }^{12} \mathrm{C}$ scaling functions for three values of $q=0.5$ (solid lines), 0.7 (dashed), and $1 \mathrm{GeV} / c$ (dotted). Initial and final states are described with the same Woods-Saxon potential. Upper panels: traditional nonrelativistic model. Middle panels: nonrelativistic model using relativistic kinematics. Lower panels: SR model.

This is illustrated in Fig. 2 for the different approaches in the shell model. There we show the same results as in Fig. 1, but presented in a different way, namely, this time plotting together the results from each of the models for the three values of $q$ simultaneously. From Fig. 2, one can appreciate that the SR approach is the only model that shows first-kind scaling. Therefore, we conclude that the relativistic corrections that are consistently accounted for in the SR, in both the current operator and the kinematics, are important for recovering first-kind scaling, although the model still lacks the dynamic ingredients from relativistic FSI needed to reproduce the shape of the experimental data, as discussed below.

Before entering into a detailed discussion of FSI, we display in Fig. 3(a) test of the SR expansion, by comparing it with a fully relativistic result. Similar tests have been carried out in the context of the relativistic Fermi gas $[1,17]$. However, at the level of the SR-WS, it is not possible to make meaningful comparisons with fully relativistic results because of the different description of the dynamics in the final states. To make a consistent comparison, one should go a step back and consider the plane-wave impulse approximation (PWIA), where the final potential is set to zero. This is done in Fig. 3, where we show the PWIA results for the $L$ and $T$ scaling functions in semi-relativistic and relativistic models. From these results, one can see that the descriptions of the initial nuclear state and of the current operator are quite similar in both models. Note that in the relativistic calculation, the $\mathrm{CC} 2$ current has been employed (see also later). The bound wave functions and energies are very close in the relativistic and nonrelativistic approaches. The small differences seen in Fig. 3 are linked to off-shell effects in the current matrix elements and are of the same order as can be found between
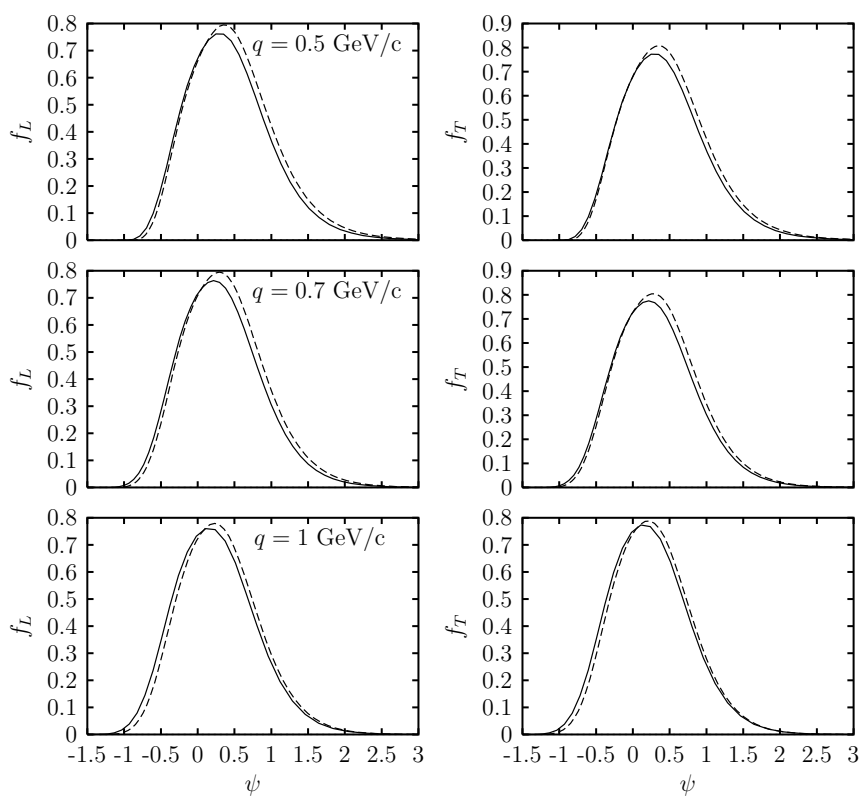

FIG. 3. Comparison of semi-relativistic and fully relativistic models in PWIA. Solid lines: SR. Dashed: relativistic with CC2 current operator.

relativistic results using different prescriptions for the current operator [41].

\section{B. FSI in the damping model}

There are two main effects embodied in the SmithWambach damping model used here: a shift and a redistribution of strength, as illustrated in Fig. 4, where we show the longitudinal response function for five values of the momentum transfer. These effects are a consequence of the folding integral in Eq. (22), which basically produces the redistribution, and of the appearance of the effective mass $M^{*}(q)$, which is responsible for the shift. However, when the energy $\omega$ is large, the parametrization of Eqs. (25) and (26) results in a small nucleon width; hence, the Lorentzian function $\rho(E, \omega)$ becomes close to a Dirac delta function and only the effective mass effect remains. This is why in Fig. 4, for the largest $q$ value, $q=1.5 \mathrm{GeV} / c$, the FSI just produce a shift of $R_{L}$. Thus, although the damping model can produce the needed effect for low-to-intermediate momentum transfers (i.e., redistribution of the strength as required by the data), for higher momentum transfer this method is basically equivalent to introducing an almost constant effective mass (of around $M^{*} / m_{N} \approx 0.8-0.9$ ).

The scaling properties of $R_{L}$ under the SWD model of FSI may be seen by examining the results in Fig. 5. There one observes that the scaling function increases with $q$; hence, scaling of the first kind is not respected by the model. Moreover it is almost symmetric, whereas the experimental data, also shown in the figure, display an asymmetry, indicating stronger redistribution of strength for positive values of $\psi^{\prime}$. The apparent incapability of the SWD to describe this feature of the data should not be considered a failure of the model, but simply an inadequate extrapolation to high energy of a model that was originally proposed to describe the damping of 

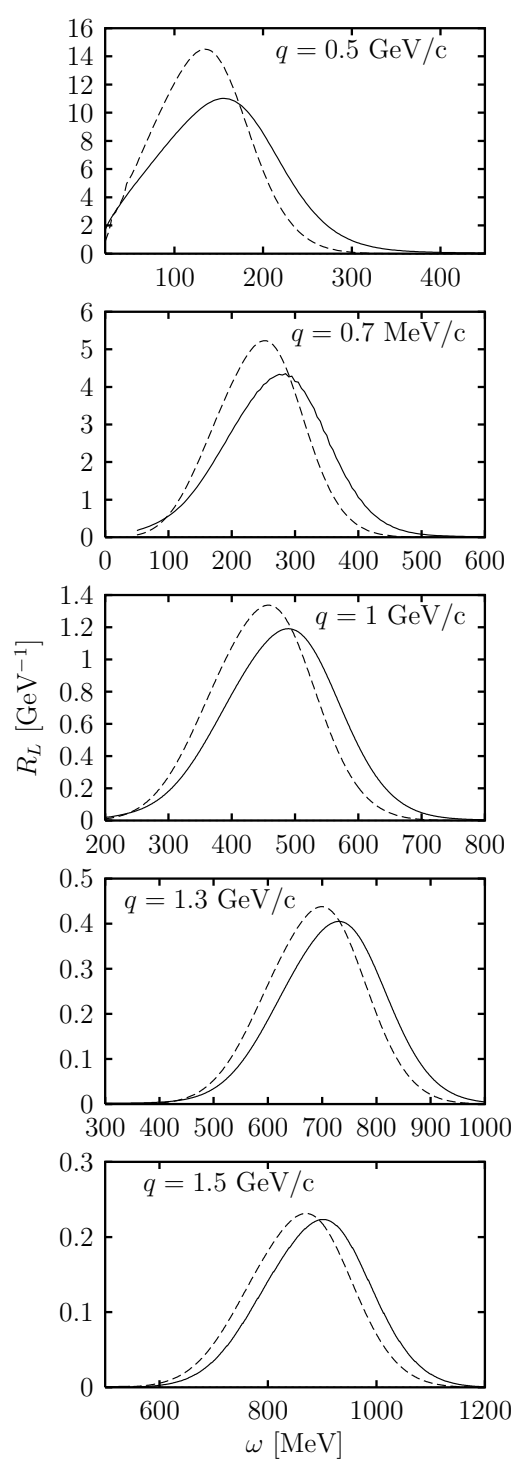

FIG. 4. Effect of FSI on longitudinal response function. Dashed lines: SR model with a Woods-Saxon potential. Solid: SR model including FSI in Smith-Wambach damping model.

the continuum nuclear response in the region of small energy transfer.

\section{DEB approach to FSI}

The second approach to FSI in this work amounts to using the DEB potential plus Darwin term as described earlier. The interest in this approach for intermediate-to-high energies originates from the results found in Refs. [41,52] where the relativistic mean-field (RMF) model was found to describe reasonably well the scaling function data. In particular, the asymmetry presented by the data appears to be obtained only in IA-based models when one uses the same form of the (real) $S$ and $V$ relativistic potential in both initial and final states. One must at this point remember that the present focus is on inclusive reactions: one should not be tempted to apply the optical potential fit to elastic data to inclusive reactions, such as $\left(e, e^{\prime}\right)$ or $\left(v_{l}, l^{-}\right)$, where different channels are open [66].

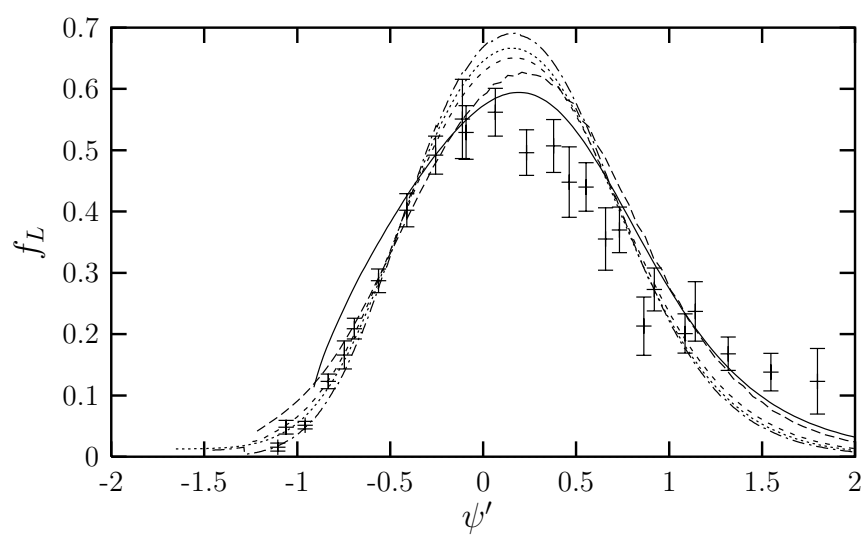

FIG. 5. Longitudinal scaling function in the SR model with Smith-Wambach damping, with several values of the momentum transfer $q=0.5$ (solid lines), 0.7 (dashed), 1.0 (short-dashed), 1.3 (dotted), and $1.5 \mathrm{GeV} / c$ (dot-dashed). No energy shift was applied to the theoretical calculation. Experimental data from Ref. [55].

It is not the purpose of this paper to find rigorous theoretical arguments to justify the use of the Hartree potential as the best choice in relativistic calculations. Developments along these lines will be discussed elsewhere [67]. We just mention here that the asymmetry found in the scaling function computed in the RMF is a consequence of the large scalar and vector parts of the potential used in the final state, which are both much smaller in the case of the real parts of typical optical potentials or in other similar approaches.

The goal of this section is to show that when conveniently implemented in the SR approach, the same relativistic Hartree potential yields results consistent with the ones obtained within the fully relativistic mean-field model. The electromagnetic response functions with the $\mathrm{DEB}+\mathrm{D}$ choice for FSI are shown in Fig. 6 for fixed momentum transfer in the range $q=0.5$ to $1.5 \mathrm{GeV} / c$. In the figure, we show the $\mathrm{SR}$ results using the Woods-Saxon potential in the final state (dashed lines) and using the DEB $+\mathrm{D}$ model of FSI (solid lines). For comparison in the same figure we also show the SR-PWIA results. As one can see, the effect of the $\mathrm{DEB}+\mathrm{D}$ model is precisely a shift and redistribution of the strength, typically what one expects to occur due to FSI mechanisms. For $q=0.5 \mathrm{GeV} / c$, the effect is similar to the SWD results of Fig. 4. However, for higher values of $q$, the effect of the FSI is maintained in the $T$ response and even increases in the case of the $L$ response, contrary to what happens in the SWD model. In this way, for $q=1.5 \mathrm{GeV} / c$, the total strength is not only redistributed but also amplified in the longitudinal response function. The behavior of $R_{L}$ for high $q$ in the high- $\omega$ tail is just a consequence of the SR expansion used for the current operator. In fact, the time component of the vector current in Eq. (10) is proportional to the $\xi_{0}$ coefficient, which in turn is proportional to the relativistic factor $\kappa / \sqrt{\tau}$ in Eq. (15). The fact that this factor becomes infinite on the light cone $\omega=q$ is just a consequence of the off-shell properties of the SR expansion, since it was performed for on-shell nucleons and therefore its applicability should be most appropriate for the QE-peak region. This is not a fault of the current, since similar 

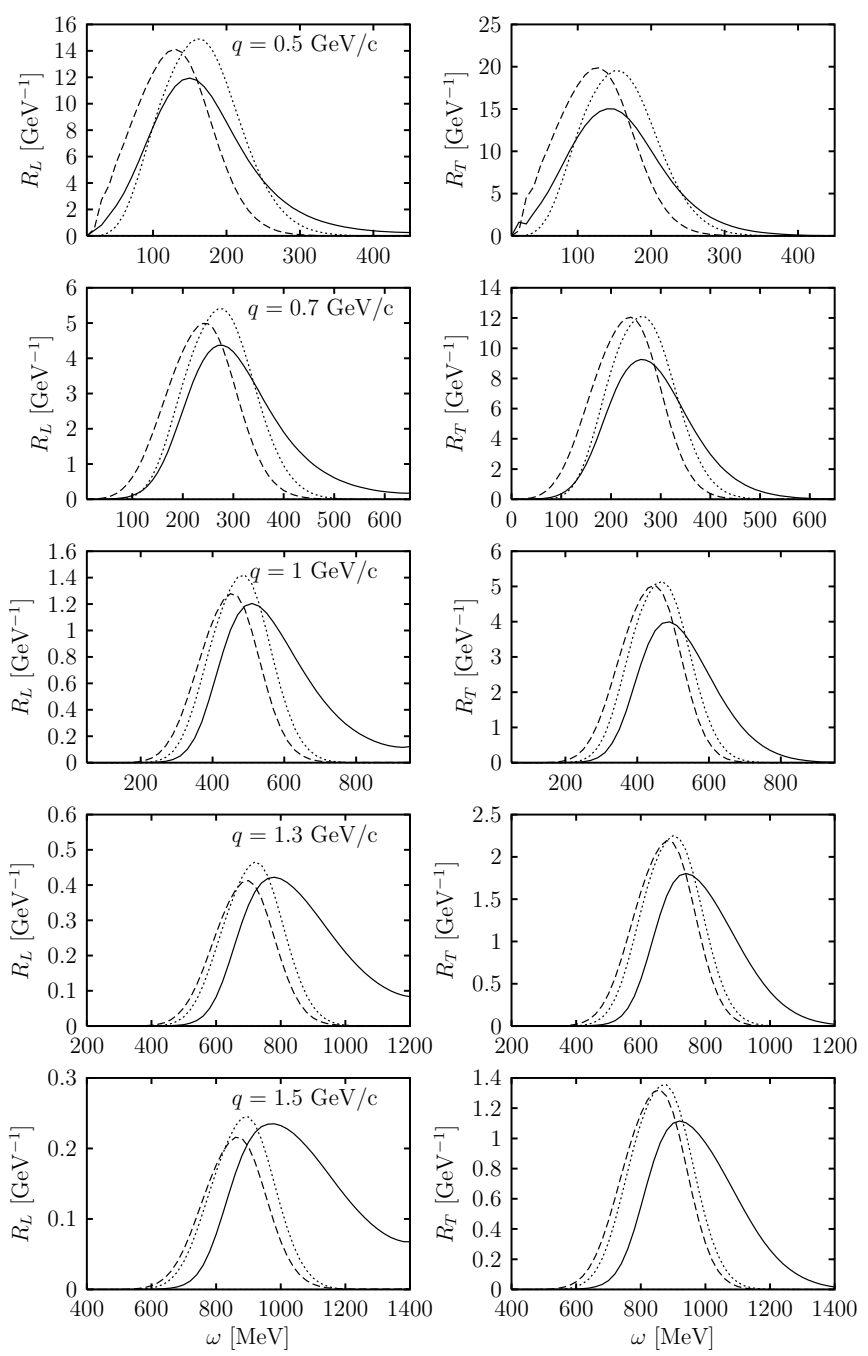

FIG. 6. Effect in the DEB $+\mathrm{D}$ model of FSI on the $L$ and $T$ responses. Dashed lines: SR-WS model. Solid: SR-DEB+D. Dotted: SR-PWIA.

anomalous off-shell effects can also be seen in prescriptions of relativistic current [41]. Therefore, one should be careful when extrapolating these operators to highly off-shell conditions, and the results of Fig. 6 should be taken with caution in the region close to $\omega=q$, in particular when $q$ is high and non-QE effects dominate the total cross section, as $R_{L}$ probably cannot be extracted experimentally there. In fact, the experimental data for the scaling function shown in this paper were taken from world data in the range $q=0.5$ to $1 \mathrm{GeV} / c$.

The interest in showing results for values of $q$ as high as $1.5 \mathrm{GeV} / c$ is related to the study of the properties of first-kind scaling performed next. In Fig. 7, we show the scaling functions in the $\mathrm{DEB}+\mathrm{D}$ model. They have been extracted from the results of Fig. 6 and are displayed as functions of the unshifted $(\psi)$ and shifted $\left(\psi^{\prime}\right)$ scaling variables. When nonzero, the energy shift is $20 \mathrm{MeV}$ in all cases. The experimental data for $f_{L}$ are shown for comparison. One can see that the $\mathrm{DEB}+\mathrm{D}$ results follow the trend of the experimental data, clearly showing the same asymmetric shape. While the strength and width of the results of Fig. 7
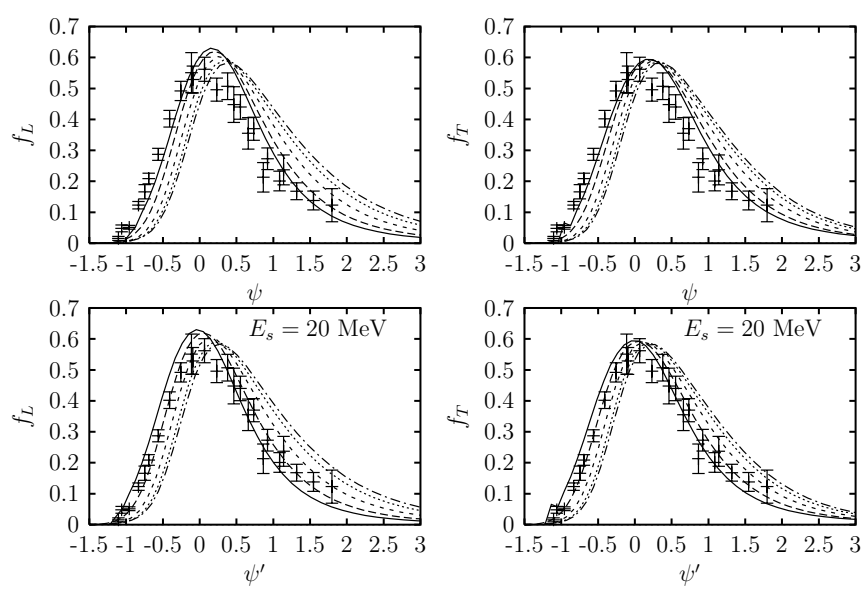

FIG. 7. First-kind scaling in the DEB $+\mathrm{D}$ model for $q=0.5,0.7$, $1.0,1.3$, and $1.5 \mathrm{GeV} / c$. Scaling functions vs $\psi$ when $E_{s}=0$ (top row) and vs $\psi^{\prime}$ when $E_{s}=20 \mathrm{MeV}$ (bottom row). Experimental data from Ref. [55].

are basically the same as the data, the theoretical results are shifted to the right with respect to the data. Though small, the shift increases with $q$. Due to the shift, one would conclude that first-kind scaling is not perfect in this model. However, note that the data do not contain values of $q$ as large as $1.5 \mathrm{GeV} / c$ and that the error bars are a consequence of a small dispersion, since scaling of the first kind is not experimentally as good as scaling of the second kind (i.e., independence of the nucleon number $A$ ). Therefore, a small scaling violation as that seen in Fig. 7 may not actually be inconsistent with the data. Another interesting conclusion from these results is that to a high degree, the $\mathrm{DEB}+\mathrm{D}$ model respects scaling of zeroth kind, i.e., $f_{L}=f_{T}$, for all $q$ values.

Another study of first-kind scaling within the $\mathrm{DEB}+\mathrm{D}$ model has been performed with results at a different kinematic setting, shown in Fig. 8. This time we show the scaling functions computed for several incident electron energies and a fixed electron scattering angle $\theta_{e}=45^{\circ}$. Here the momentum transfer is not constant and changes with $\psi$. As a consequence, the scaling works better and the curves are closer to each other.

At this point, a test of the present SR model is possible by comparing it with a fully relativistic model. Since the final states in the DEB $+\mathrm{D}$ approach are computed essentially as in the RMF approach presented in Refs. [41,52], both models should give similar results if the relevant relativistic corrections are included properly in the SR approach. A first
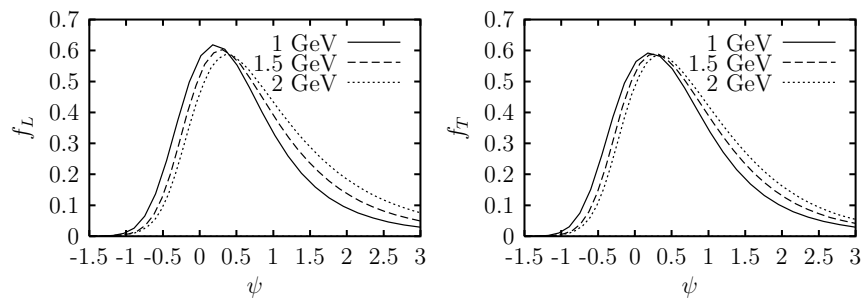

FIG. 8. First-kind scaling in the DEB $+\mathrm{D}$ model for incident electron energies $\epsilon_{e}=1,1.5$, and $2 \mathrm{GeV}$. Electron scattering angle $\theta_{e}=45^{\circ}$, and $E_{s}=0$. 

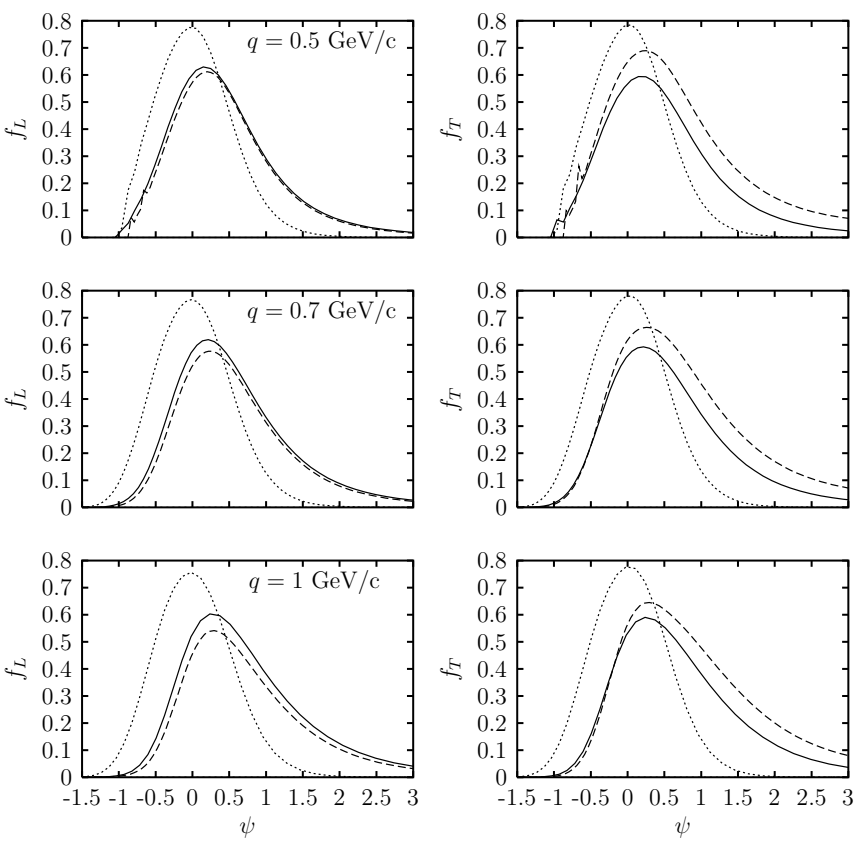

FIG. 9. Electromagnetic scaling functions for three values of the momentum transfer. Dotted lines: SR model with a Woods-Saxon potential in the final state. Solid: SR model using the $D E B+D$ approach for FSI. Dashed: RMF with the CC2 current operator. Here $E_{s}=0$.

comparison is performed in Fig. 9 for three values of the momentum transfer. The RMF results are computed with the CC2 current operator. The SR model using the Woods-Saxon potential in the final state is also shown for comparison. It is apparent from the figure that the behavior of the SR-DEB+D results is similar to that in the fully relativistic approach. The differences seen between the two models can partially be attributed to the peculiar off-shell properties of the $\mathrm{CC} 2$ and SR currents, as well as to the four-component structure of the wave functions involved in the RMF model compared with the SR-DEB+D. In fact, differences of the same order can also be found in the RMF approach between results obtained using other current prescriptions. An example is shown in Fig. 10, where we compare our results for $q=1 \mathrm{GeV} / c$ with the RMF results employing two current prescriptions. For this value of the momentum transfer, the $\mathrm{DEB}+\mathrm{D}$ model gives similar results to those for the RMF model with the CC3 current operator, although not exactly the same. From the results of Figs. 9
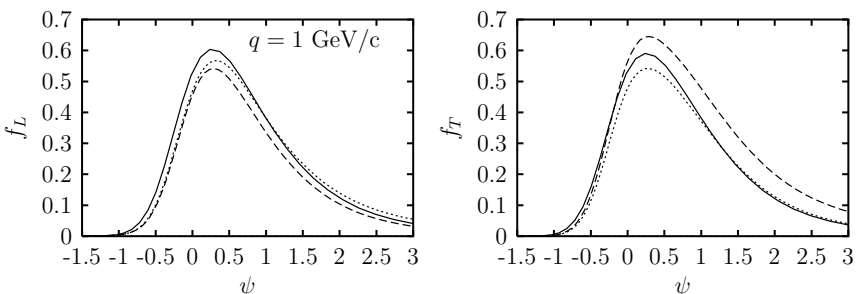

FIG. 10. Electromagnetic scaling functions for $q=1 \mathrm{GeV} / c$. Solid lines: SR model within the DEB+D approach for FSI. Dashed: RMF with the CC2 current operator. Dotted: RMF with the CC3 current operator. and 10 we conclude that our model contains the relevant relativistic corrections in this kinematic region. Our current operator could then be considered at this level as another prescription for off-shell extrapolation of the relativistic nucleon current. At this point, it is also interesting to note that the RMF model with the $\mathrm{CC} 2$ operator leads to a clear violation of zeroth-kind scaling (likewise for $\mathrm{CC} 1$, but not for CC3), contrary to what happens with SR-DEB+D.

Motivated by the results of Fig. 7, where first-kind scaling is fulfilled except for a small shift, and in order to explore further the scaling properties of our model, we have performed the calculation of the scaling variable $\psi^{\prime}$ using a $q$-dependent energy shift $E_{S}(q)$. The value of $E_{s}(q)$ is fitted to obtain the maximum of $f_{L}$ at $\psi^{\prime}=0$. The results of this fit, shown in Fig. 11, indicate that the collapse of the curves is improved by this procedure. Although the scaling is not perfect, it is remarkable that our results give essentially the same scaling function for a wide range of $q$ values $(0.5-1.5 \mathrm{GeV} / c)$. The scaling is better for the transverse function $f_{T}$, while the width of $f_{L}$ slightly increases with $q$. In Fig. 11, we also show a comparison with the experimental data. The agreement between theory and experiment is excellent. We recall that data refer only to the analysis of the longitudinal response, hence caution should be exercised when comparing the calculated transverse scaling function $f_{T}$ and data. Finally, we also present in Fig. 11 the shift energy $E_{s}(q)$ as a function of $q$, showing that the dependence of the fit is clearly linear. This linear behavior of $E_{s}(q)$ is connected to the energy dependence of the DEB potential, which is also linear $[30,60]$. We illustrate this dependence in Fig. 12, where we plot the
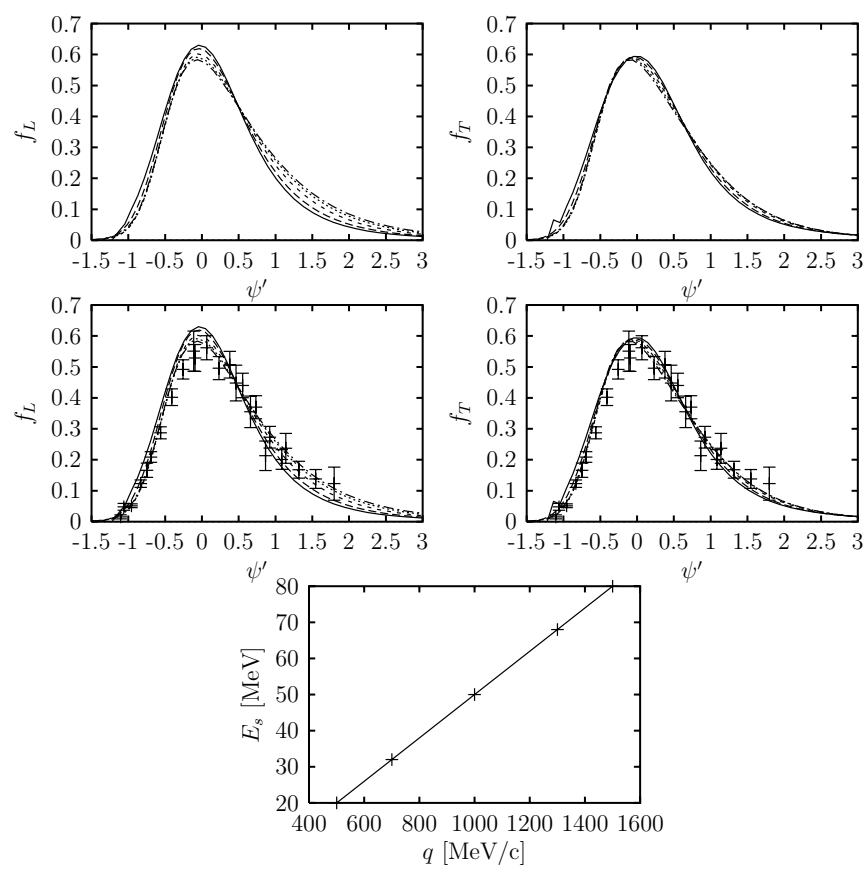

FIG. 11. First-kind scaling in the SR-DEB+D model for $q=0.5$, $0.7,1.0,1.3$, and $1.5 \mathrm{GeV} / c$. First row: Scaling functions vs $\psi^{\prime}$ using a $q$-dependent energy shift $E_{s}(q)$. Second row: same results compared with experimental data [55]. Bottom panel: linear behavior of $E_{s}(q)$. Fitted values of $E_{s}$ are 20,32, 50, 68, and $80 \mathrm{MeV}$. 

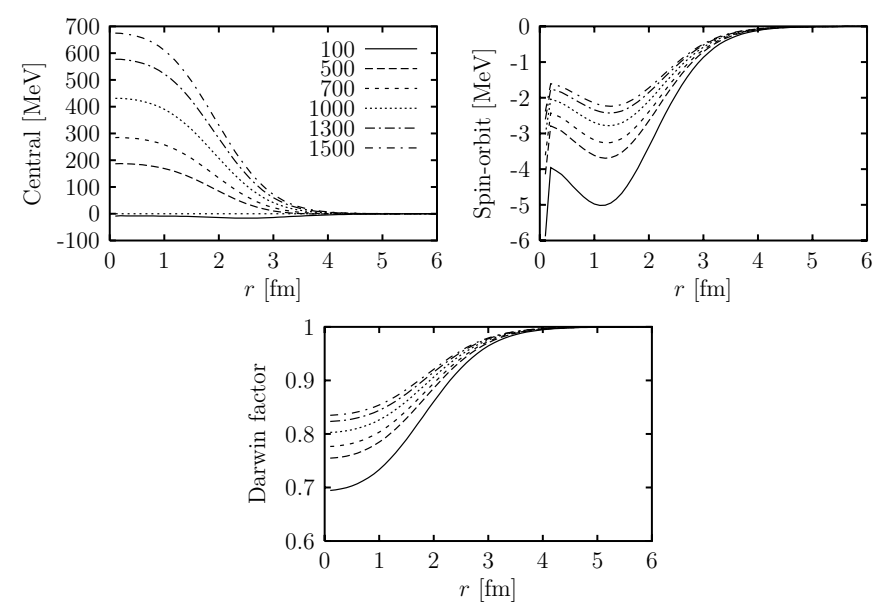

FIG. 12. Top row: DEB potential for final nucleon kinetic energies 100-1500 MeV. Bottom panel: same for the Darwin factor.

central and spin-orbit parts of the DEB potential for a range of final nucleon kinetic energies from 100 to $1500 \mathrm{MeV}$. More details on the properties of the DEB potential can be found in Ref. [30]. From Fig. 12, it is evident that the central part of the DEB potential increases linearly with the energy and that it is very repulsive for high energy. The spin-orbit part, on the other hand, is rather small and unimportant for these energies. In the same figure, we also show the Darwin factor $K(r, E)$ for the same nucleon energies.

The highly repulsive behavior of the DEB potential is the main reason for the strength redistribution seen in our results. A repulsive potential favors the emission of nucleons having high enough available energy. Accordingly, more strength is placed in the high energy tail of the cross section or, equivalently, at positive values of $\psi^{\prime}$ in the scaling function. One should mention here that the DEB potential alone is not enough to produce reasonable results for the scaling function. The Darwin factor $K(r, E)$, shown in the last panel of Fig. 12, must also be applied to the wave function. We illustrate this point in Fig. 13, where we compare the SR-WS model (namely, with Woods-Saxon potential in the initial and final states) with the SR-DEB, but with the Darwin term set to unity. One can see that the DEB potential alone produces the mentioned shift and widening of the scaling function to higher energies, because of the repulsive character of the potential, although the strength is still too high when compared with the data.

The effect of the Darwin term can be appreciated in Fig. 14, where we plot the scaling functions in the SR-DEB approach with and without that factor (or equivalently by setting $K(r, E)=1$ in the calculation). Since $K(r)<1$ inside the nucleus, its effect is a reduction of the scaling function. This reduction is precisely the one needed to reach the experimental data, also shown in Fig. 14. Thus the Darwin factor is essential in this approach to FSI.

One should mention that the full DEB $+\mathrm{D}$ model is not equivalent to solving a Schrödinger equation with a Hermitian local potential. Actually, the wave function in Eq. (27) is a solution of such an equation with a nonlocal potential, since apart from the dependence on the energy $E$, dependence on the momentum or gradient operator also appears from the
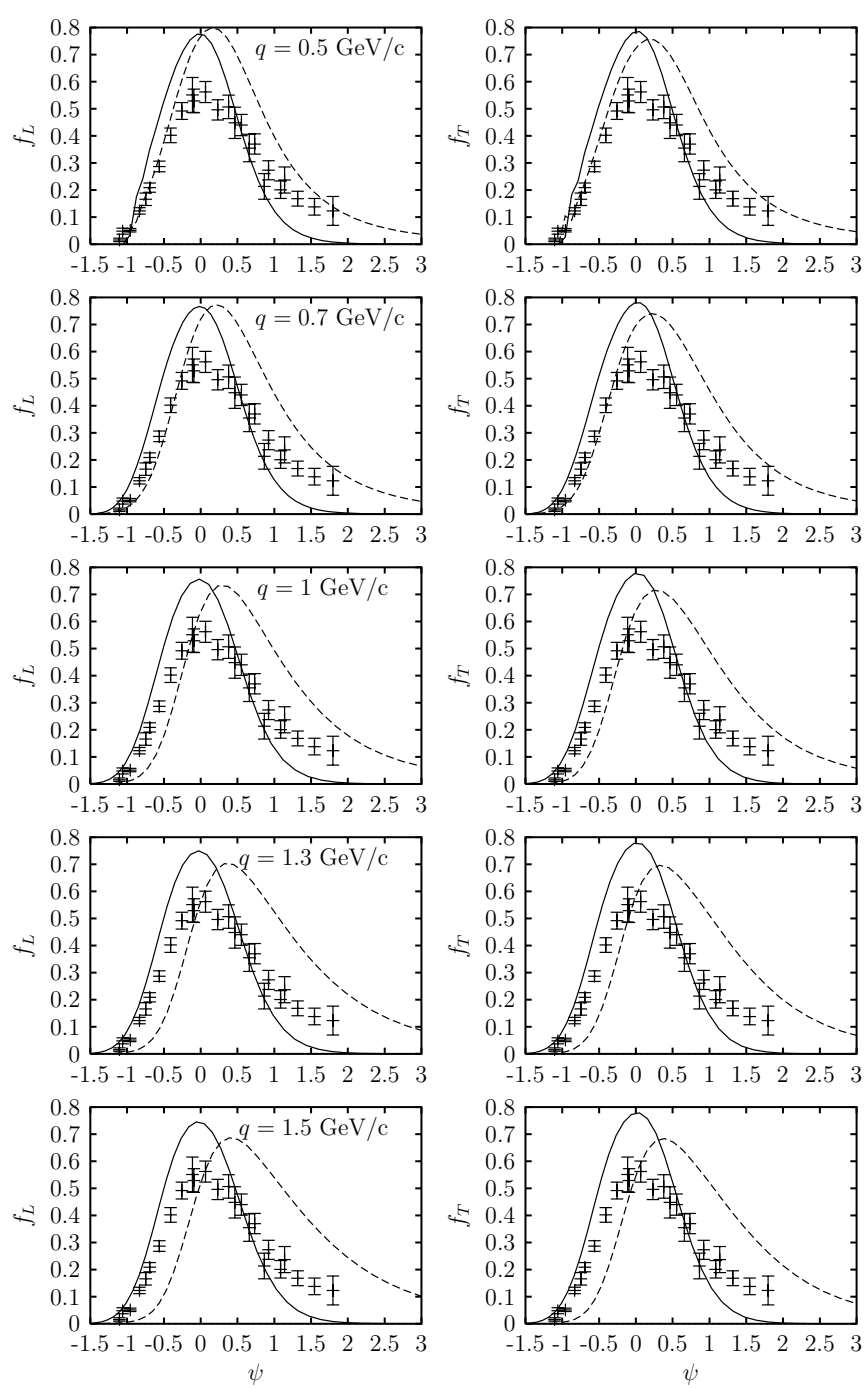

FIG. 13. Effect of DEB potential without the Darwin term. Solid lines: SR model with the same Woods-Saxon potential in the initial and final states. Dashed: SR-DEB results with the Darwin term set to unity. Experimental data from Ref. [55].

reduction of the Dirac equation [30,56,57,60]. This means that the solution of the Schrödinger equation with DEB potential plus Darwin factor is not comparable to any other nonrelativistic or relativized solutions obtained with local potentials. The nonlocality contained in the $\mathrm{DEB}+\mathrm{D}$ approach introduced here, also implicit in the Dirac equation and made explicit by means of the $\mathrm{DEB}+$ Darwin reduction, thus becomes essential in producing the kind of effects that usually appear in nonrelativistic approaches of FSI related to the role of correlations and/or exchange terms in the optical potentials. In particular, one could argue that in the SWD model discussed above, the inclusion of $2 \mathrm{p}-2 \mathrm{~h}$ intermediate states gives rise also to nonlocal effects in the final state.

A better appreciation of the effect of the DEB potential without the Darwin factor can be seen in Fig. 15, where we compare the scaling functions in the SR-WS and SR-DEB potentials. Once again the Darwin factor is set to unity. The $q$-dependent shift $E_{s}(q)$ has been applied to the DEB results. 

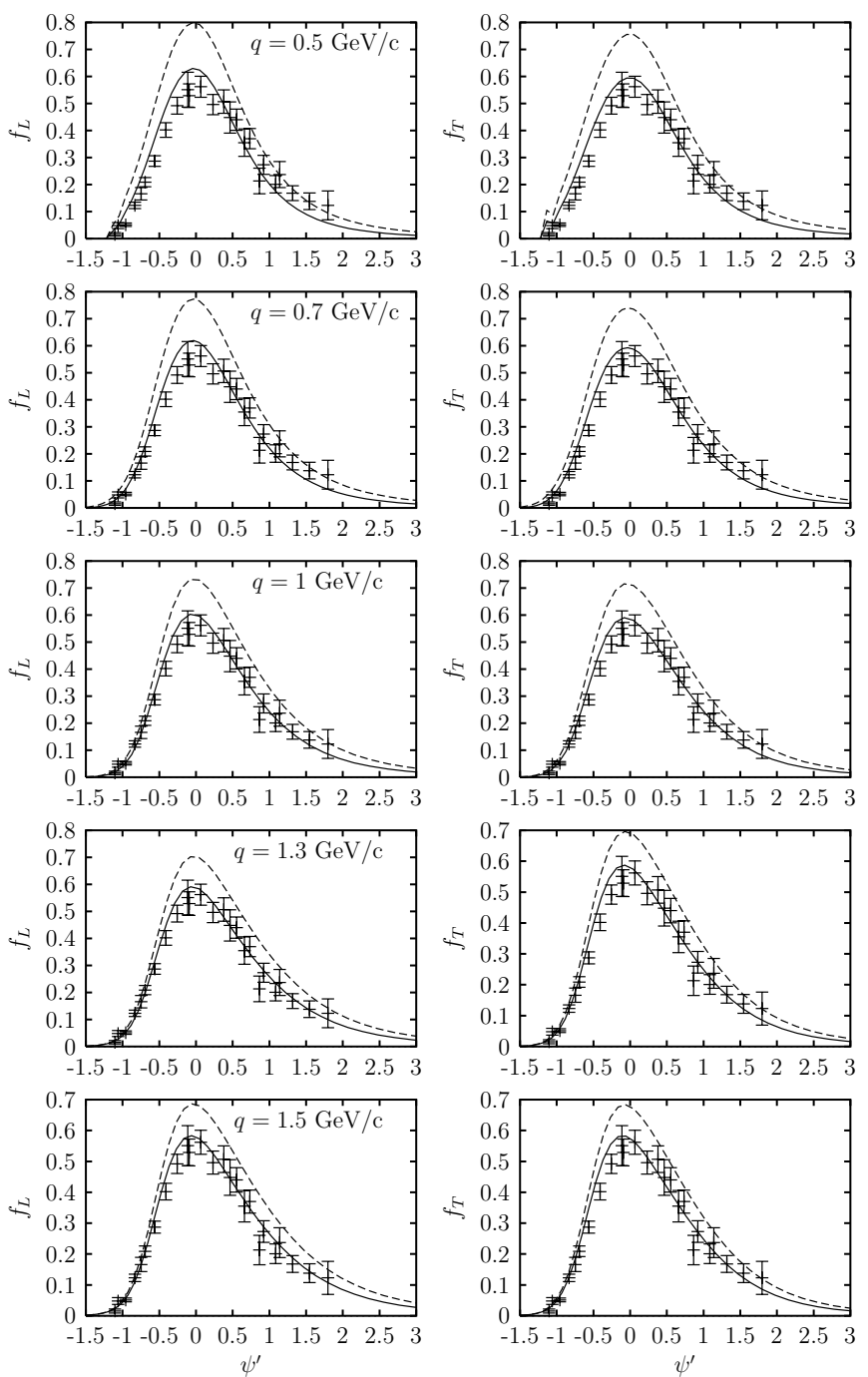

FIG. 14. Effect of Darwin term on scaling function in the SR model. Solid lines: total DEB+D result. Dashed: results with the Darwin term set to one. Experimental data from Ref. [55].

The widening of the distribution to higher energies, producing a longer tail, is clear. Note also that the DEB potential does not modify the results in the region $\psi^{\prime}<0$, and that there is a reduction of the maximum in the region $\psi^{\prime} \sim 0$ (the magnitude of the reduction increases with $q$ ).

\section{Applications to neutrino reactions}

We now exploit the SR-DEB+D model introduced in the last section to make predictions for QE CC neutrino reactions. Given the success of that model in describing the $\left(e, e^{\prime}\right)$ superscaling data, the neutrino cross section results obtained with our model can be considered at least reasonable in a kinematic range from intermediate-to-high energies and momentum transfers.

A selection of results for the ${ }^{12} \mathrm{C}\left(v_{\mu}, \mu^{-}\right)$differential $\mathrm{QE}$ cross section are presented in Figs. 16 and 17, for incident neutrino energies of 1 and $1.5 \mathrm{GeV}$, respectively. We show results for a range of scattering angles from $15^{\circ}$ to $150^{\circ}$. All of the results in the figures have been obtained with the SR
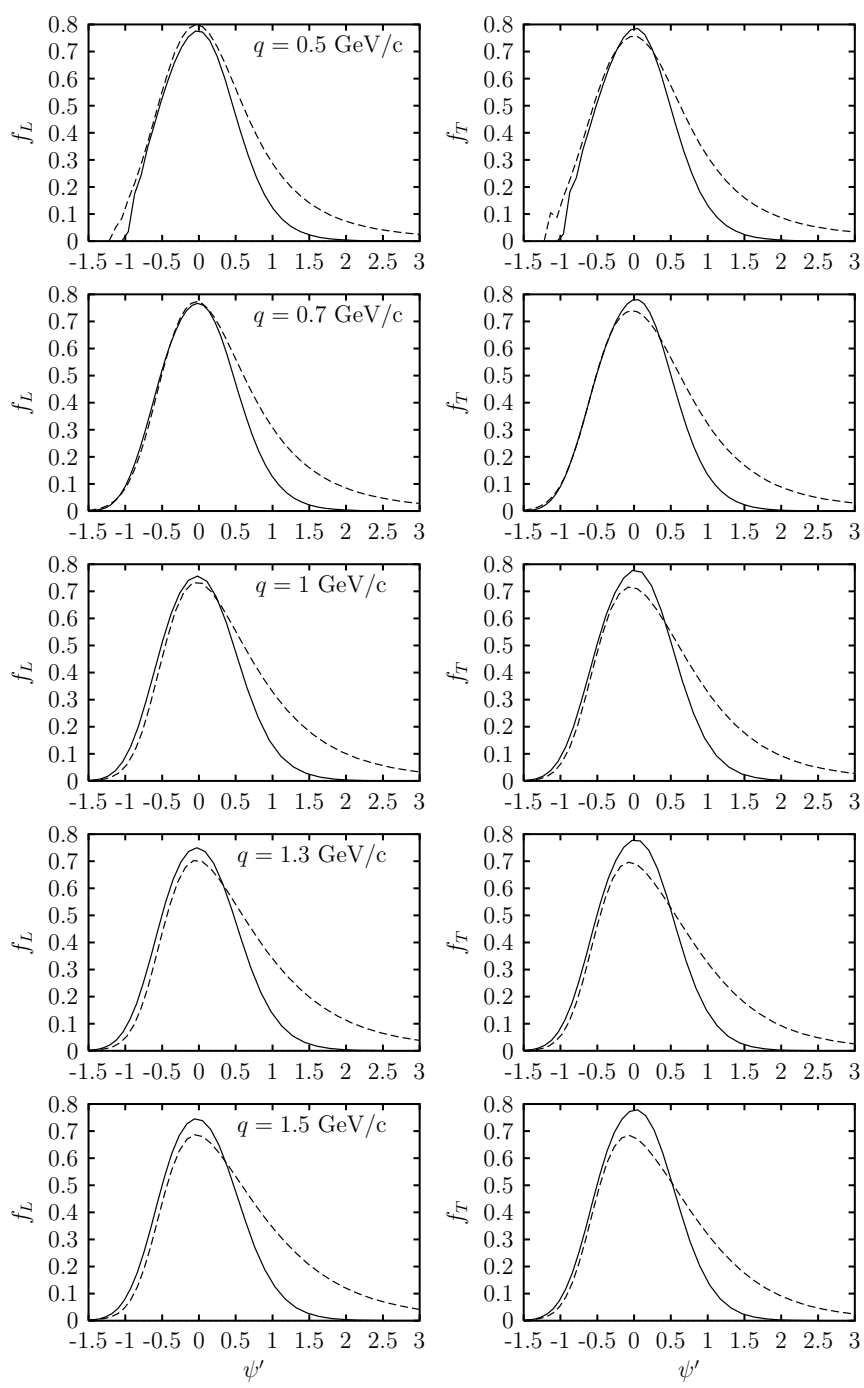

FIG. 15. Effect of DEB potential without Darwin term. Solid lines: SR-WS. Dashed: SR-DEB with the Darwin term set to unity and with the $q$-dependent energy shift $E_{s}(q)$.

model with different ingredients for the FSI. The results within the DEB $+\mathrm{D}$ model of FSI are shown with solid lines, while the dotted lines are the SR-WS results using the same WoodsSaxon potential in initial and final states. We display the cross section as a function of the final muon energy $\epsilon^{\prime}$. Therefore, the tail produced by the FSI can now be seen to the left of the peak of the SR-WS results. Finally, with dashed lines we show the results obtained in a third calculation which closely follows the general procedure originally introduced in Ref. [61] and referred to as the SuSA approach.

Just as when the SuSA approach was performed using data, here the theoretical $\left(e, e^{\prime}\right)$ cross section is divided by the single-nucleon factor to obtain the scaling function, which in turn is multiplied by the relevant $\mathrm{CC}$ weak factor, to obtain the neutrino cross section. That is, this procedure is similar to the SuSA approach in Ref. [61] except that instead of using the "experimental" superscaling function obtained from the analysis of $\left(e, e^{\prime}\right)$ world data, in this case we make use of the scaling function derived from $\left(e, e^{\prime}\right)$ results with 

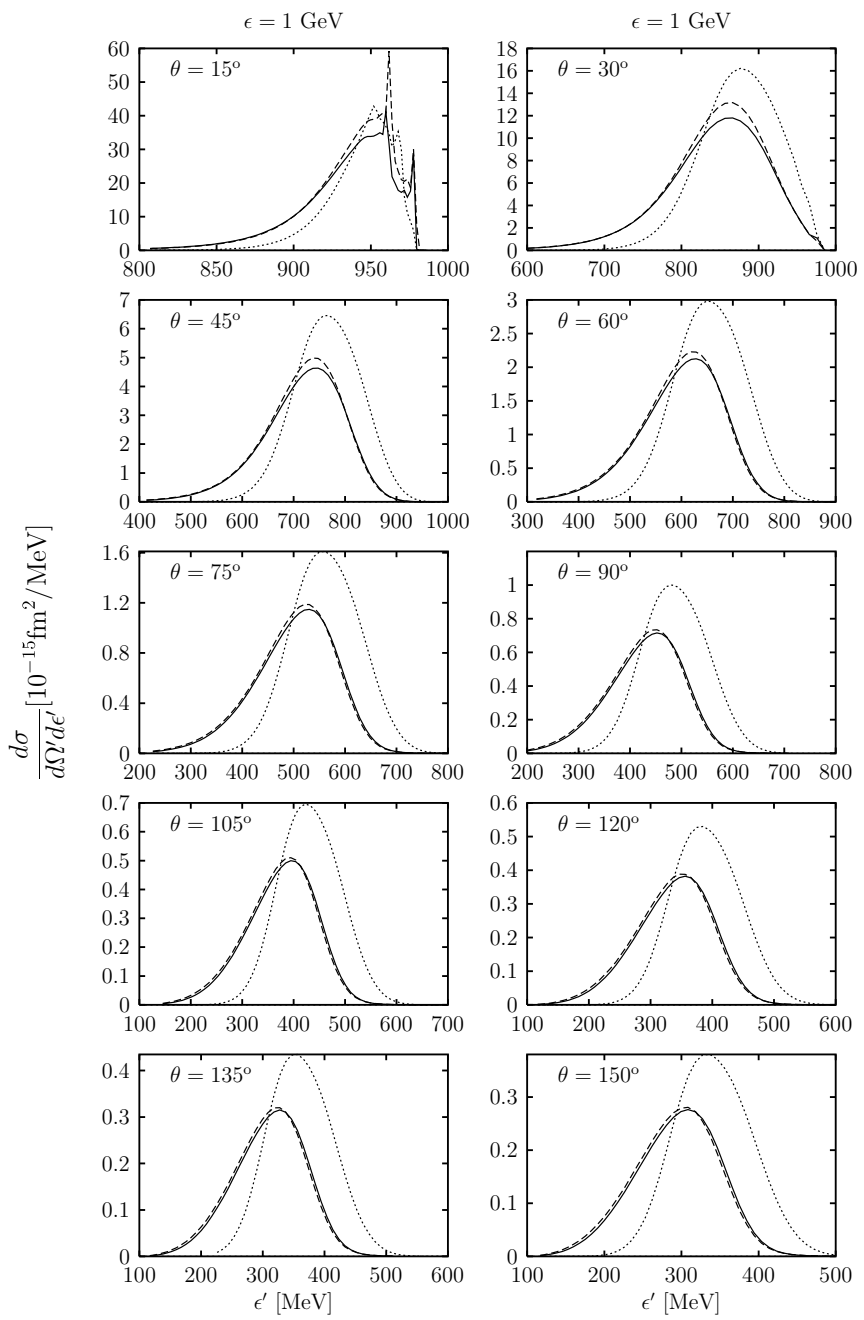

FIG. 16. Double-differential cross section for the ${ }^{12} \mathrm{C}\left(v_{\mu}, \mu^{-}\right)$ reaction for incident neutrinos of $1 \mathrm{GeV}$, computed with several versions of SR model. Dotted lines: Woods-Saxon potential. Solid: $\mathrm{DEB}+\mathrm{D}$. Dashed: reconstructed from the $\left(e, e^{\prime}\right) \mathrm{DEB}+\mathrm{D}$ model using the SuSA.

the SR-DEB+D model. Note that the reconstruction of the $\left(v_{\mu}, \mu^{-}\right)$cross section from the $\left(e, e^{\prime}\right)$ data (SuSA) or $\left(e, e^{\prime}\right)$ calculations (we denote it as superscaling-based approach to avoid confusion with SuSA which implies the "experimental" scaling function) assumes that the scaling function for both reactions is similar for the kinematics of interest. Thus in Figs. 16 and 17, we treat the solid lines of the DEB+D model as "exact" and check the accuracy of the superscaling-based approach by comparing the dashed lines with the solid ones. In general, the superscaling-based results are very close to the $\mathrm{DEB}+\mathrm{D}$ results. The differences between the two sets of results become smaller as the scattering angle and neutrino energy increase, since bigger values of the momentum transfer are involved. The biggest differences appear for very small angles, implying rather small values of the momentum transfer. In these cases, the description of the giant resonance region needs a nuclear model containing collective degrees of freedom such as the random-phase approximation (RPA), which is not included in our approach. An example is shown
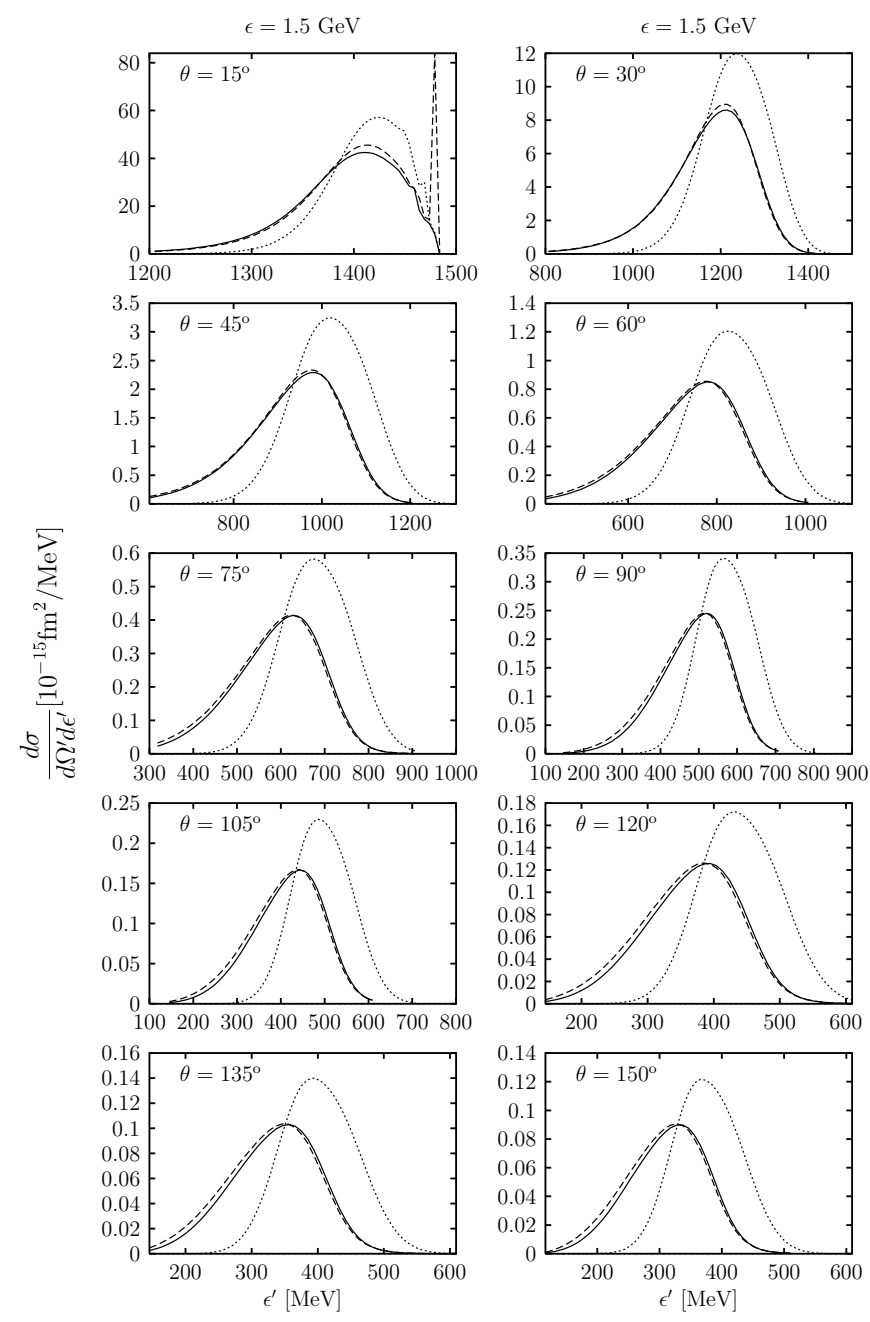

FIG. 17. Same as Fig. 16, but for neutrinos of $1.5 \mathrm{GeV}$.

for $\theta=15^{\circ}$ in Figs. 16 and 17. The presence of the narrow peaks appearing as potential resonances clearly violates the scaling of the cross section in that region. However, for higher values of the scattering angle, the superscaling-based analysis begins to be applicable, and its predictions can be considered quite reasonable, with an error typically below $10 \%$.

\section{CONCLUSIONS}

In this work, we have presented improvements over the semi-relativistic approach to electron and neutrino reactions for intermediate-to-high energies and momentum transfers in the $\mathrm{QE}$ region. Going a step beyond the continuum shell model, we have explored two approaches to describing the FSI: the Smith-Wambach damping model and the Dirac-equationbased potential. The former is applicable to low energies and has simply been extrapolated here to relativistic energies, while the latter is more appropriate for the kinematic regime considered in this paper and has been our main focus.

Thus, in addition to using the semi-relativistic expansion of the nucleon current in powers of $p / m_{N}$ and relativistic kinematics, we have used the DEB form of the relativistic Hartree potential term to describe the continuum wave function 
of the ejected nucleon. Furthermore, we have included in the wave function the nonlocalities arising from the reduction of the Dirac equation by multiplying it by the corresponding Darwin factor.

First, we focused on the analysis of the electromagnetic response functions. In particular, we investigated the properties of scaling of the first kind displayed by our results. The longitudinal and transverse scaling functions were computed for a wide range of momentum transfers, from 0.5 to $1.5 \mathrm{GeV}$. Several aspects and details of the different theoretical ingredients embodied in our model were analyzed. In particular, we examined the effects of the relativistic corrections, and we presented comparisons with fully relativistic results.

We have found that our model approximately fulfills firstkind scaling except for a small energy shift, $E_{s}(q)$, which turns out to be linear in $q$. This behavior has been connected with the repulsive character of the DEB potential, which also depends linearly on the nucleon energy. The Darwin factor has been found to be essential for the description of the experimental scaling function data. The Darwin factor is needed to correct for mathematically unavoidable nonlocalities arising in the differential equation describing the upper component of the relativistic nucleon wave function.

Is is a remarkable result of this work that, except for the energy shift, our model gives essentially the same scaling function for a $1 \mathrm{GeV}$ wide range of three-momenta $q-$ spanning a kinematic region that extends from nonrelativistic to relativistic conditions - and that the behavior of the theoretical scaling function is essentially the same as that of the experimental data. The study of the theoretical energy shift performed here can be of help for future analyses of the scaling properties of experimental data. In particular, it suggests that the small scaling violations of the first kind found in the $\left(e, e^{\prime}\right)$ data can be used to extract valuable information about the strength of the FSI.

Finally, we have presented an application of the model to the inclusive $\mathrm{CC}$ neutrino reaction ${ }^{12} \mathrm{C}\left(v_{\mu}, \mu^{-}\right)$in the region of the $\mathrm{QE}$ peak. Results have been presented in a range of kinematics for several neutrino energies and muon scattering angles. In particular, we used our model to investigate theoretically the kinematic range of validity of the superscaling approach in reconstructing the neutrino cross section from the electromagnetic scaling function. The validity of the superscaling-based approach has been verified with our model for kinematics involving values of the momentum transfer large enough such that the QE region is not contaminated by the presence of giant resonances. For incident neutrinos of $1 \mathrm{GeV}$, this happens for angles typically bigger than $\sim 15^{\circ}$. In these cases, within our model, the superscaling-based approach is seen to be very successful in being able to reconstruct the neutrino cross section from the electromagnetic one.

\section{ACKNOWLEDGMENTS}

This work was partially supported by funds provided by DGI (Spain) and FEDER funds, under Contracts Nos. BFM2002-03218, FIS05-01105, FPA2005-04460, FPA200613807, and FPA2006-07393, by the Junta de Andalucía, by the Comunidad de Madrid and UCM, and by the INFNCICYT Collaboration agreement (project "Study of Relativistic Dynamics in Electron and Neutrino Scattering"). T.W.D. also acknowledges support from the U.S. Department of Energy under cooperative research agreement No. DE-FC0294ER40818. We thank the support of INT Program 06-2b, "Neutrino Response Functions from Threshold to $10 \mathrm{GeV}$."
[1] J. E. Amaro, J. A. Caballero, T. W. Donnelly, E. Moya de Guerra, A. M. Lallena, and J. M. Udias, Nucl. Phys. A602, 263 (1996).

[2] S. Jeschonnek and T. W. Donnelly, Phys. Rev. C 57, 2438 (1998).

[3] J. M. Udias, J. A. Caballero, E. Moya de Guerra, J. E. Amaro, and T. W. Donnelly, Phys. Rev. Lett. 83, 5451 (1999).

[4] J. E. Amaro, M. B. Barbaro, J. A. Caballero, T. W. Donnelly, and A. Molinari, Phys. Rep. 368, 317 (2002).

[5] M. Mazziotta, J. E. Amaro, and F. A. de Saavedra, Phys. Rev. C 65, 034602 (2002).

[6] J. E. Amaro, M. B. Barbaro, J. A. Caballero, and F. Kazemi Tabatabaei, Phys. Rev. C 68, 014604 (2003).

[7] S. Boffi, F. Capuzzi, P. Demetriou, and M. Radici, Nucl. Phys. A637, 585 (1998).

[8] J. Adam and H. Arenhovel, Nucl. Phys. A614, 289 (1997).

[9] S. Frullani and J. Mougey, Adv. Nucl. Phys. 14, 1 (1984).

[10] C. Giusti and F. D. Pacati, Nucl. Phys. A336, 427 (1980).

[11] A. M. Saruis, Phys. Rep. 235, 57 (1993).

[12] G. Orlandini and M. Traini, Rep. Prog. Phys. 54, 257 (1991).

[13] J. E. Amaro, J. A. Caballero, T. W. Donnelly, and E. Moya de Guerra, Nucl. Phys. A611, 163 (1996).

[14] J. D. Walecka, Theoretical Nuclear and Subnuclear Physics (Oxford University, New York, 1995).

[15] J. E. Amaro, M. B. Barbaro, J. A. Caballero, T. W. Donnelly, and A. Molinari, Nucl. Phys. A643, 349 (1998).
[16] J. E. Amaro, M. B. Barbaro, J. A. Caballero, T. W. Donnelly, and A. Molinari, Nucl. Phys. A657, 161 (1999).

[17] J. E. Amaro, M. B. Barbaro, J. A. Caballero, T. W. Donnelly, and C. Maieron, Phys. Rev. C 71, 065501 (2005).

[18] Y. Fukuda et al. (Super-Kamiokande Collaboration), Phys. Rev. Lett. 81, 1562 (1998).

[19] M. H. Ahn et al. (K2K Collaboration), Phys. Rev. Lett. 90, 041801 (2003).

[20] E. Ables et al. (MINOS Collaboration), Fermilab Proposal 0875 (unpublished).

[21] E. Church et al., (BooNe Collaboration), Fermilab Proposal 0898 (unpublished)

[22] I. Ambats et al. (NOvA Collaboration), Fermilab Proposal 0929 (unpublished).

[23] D. Drakoulakos et al. (MINERvA Collaboration), Fermilab Proposal 0938 (unpublished).

[24] R. Gran, talk given at the INT Program 06-2b, "Neutrino Response Functions from Threshold to 10 GeV," July 31August 25, 2006 (unpublished). http://www.int.washington.edu/ talks/WorkShops/int_06_2b/

[25] G. P. Zeller, talk given at the INT Program 06-2b, "Neutrino Response Functions from Threshold to $10 \mathrm{GeV}$," July 31August 25, 2006 (unpublished). http://www.int.washington.edu/ talks/WorkShops/int_06_2b/ 
[26] G. P. Zeller, Nucl. Phys. B, Proc. Suppl. 155, 111 (2006).

[27] A. Picklesimer and J. W. Van Orden, Phys. Rev. C 35, 266 (1987).

[28] A. Picklesimer and J. W. Van Orden, Phys. Rev. C 40, 290 (1989).

[29] J. M. Udías, P. Sarriguren, E. Moya de Guerra, E. Garrido, and J. A. Caballero, Phys. Rev. C 48, 2731 (1993).

[30] J. M. Udías, P. Sarriguren, E. Moya de Guerra, E. Garrido, and J. A. Caballero, Phys. Rev. C 51, 3246 (1995).

[31] W. M. Alberico, M. B. Barbaro, S. M. Bilenky, J. A. Caballero, C. Giunti et al., Nucl. Phys. A623, 471 (1997).

[32] W. M. Alberico, M. B. Barbaro, S. M. Bilenky, J. A. Caballero, C. Giunti et al., Phys. Lett. B438, 9 (1998).

[33] M. C. Martinez, P. Lava, N. Jachowicz, J. Ryckebusch, K. Vantournhout, and J. M. Udias, Phys. Rev. C 73, 024607 (2006).

[34] Y. Jin, D. S. Onley, and L. E. Wright, Phys. Rev. C 45, 1333 (1992).

[35] K. S. Kim and L. E. Wright, Phys. Rev. C 68, 027601 (2003).

[36] K. S. Kim and L. E. Wright, Phys. Rev. C 72, 064607 (2005).

[37] C. R. Chinn, A. Picklesimer, and J. W. Van Orden, Phys. Rev. C 40, 790 (1989).

[38] A. Meucci, F. Capuzzi, C. Giusti, and F. D. Pacati, Phys. Rev. C 67, 054601 (2003).

[39] A. Meucci, C. Giusti, and F. D. Pacati, Nucl. Phys. A739, 277 (2004).

[40] C. Maieron, M. C. Martinez, J. A. Caballero, and J. M. Udias, Phys. Rev. C 68, 048501 (2003); Nucl. Phys. B139, 226 (2005).

[41] J. A. Caballero, Phys. Rev. C 74, 015502 (2006).

[42] A. Fabrocini and S. Fantoni, Nucl. Phys. A503, 375 (1989).

[43] G. Co', K. Q. Quader, R. Smith, and J. Wambach, Nucl. Phys. A485, 61 (1988).

[44] J. E. Amaro, G. Co‘, E. M. V. Fasanelli, and A. M. Lallena, Phys. Lett. B277, 249 (1992).

[45] J. E. Amaro, G. Co', and A. M. Lallena, Nucl. Phys. A578, 365 (1994).

[46] A. De Pace and M. Viviani, Phys. Rev. C 48, 2931 (1993).

[47] S. Jeschonnek, A. Szczurek, G. Co“, and S. Krewald, Nucl. Phys. A570, 599 (1994).

[48] A. Gil, J. Nieves, and E. Oset, Nucl. Phys. A627, 543 (1997).
[49] R. D. Smith and J. Wambach, Phys. Rev. C 38, 100 (1988).

[50] A. Botrugno and G. Co', Nucl. Phys. A761, 200 (2005).

[51] J. E. Amaro, A. M. Lallena, and J. Nieves, Nucl. Phys. A623, 529 (1997).

[52] J. A. Caballero, J. E. Amaro, M. B. Barbaro, T. W. Donnelly, C. Maieron, and J. M. Udias, Phys. Rev. Lett. 95, 252502 (2005).

[53] T. W. Donnelly and I. Sick, Phys. Rev. Lett. 82, 3212 (1999).

[54] T. W. Donnelly and I. Sick, Phys. Rev. C 60, 065502 (1999).

[55] C. Maieron, T. W. Donnelly, and I. Sick, Phys. Rev. C 65, 025502 (2002).

[56] L. G. Arnold, B. C. Clark, R. L. Mercer, and P. Schwandt, Phys. Rev. C 23, 1949 (1981); B. C. Clark, L. G. Arnold, R. L. Mercer, D. A. Hutcheon, J. M. Cameron, R. P. Liljestrand et al., Bull. Am. Phys. Soc. 26, 567 (1981); L. G. Arnold, B. C. Clark, and R. L. Mercer, Phys. Rev. C 23, 15 (1981); B. C. Clark, L. G. Arnold, and R. L. Mercer, Bull. Am. Phys. Soc. 25, 520 (1980); 25, 741 (1980); L. G. Arnold, B. C. Clark, and R. L. Mercer, Phys. Rev. C 19, 917 (1979); L. G. Arnold and B. C. Clark, Phys. Lett. B84, 46 (1979).

[57] M. Jaminon, C. Mahaux, and P. Rochus, Phys. Rev. C 22, 2027 (1980).

[58] C. Bleve, G. Co', I. De Mitri, P. Bernardini, G. Mancarella, D. Martello, and A. Surdo, Astropart. Phys. 16, 145 (2001).

[59] G. Co', C. Bleve, I. De Mitri, and D. Martello, Nucl. Phys. B112, 210 (2002).

[60] J. M. Udias, J. R. Vignote, E. Moya de Guerra, A. Escuderos, and J. A. Caballero, in Proceedings of the 5th Workshop on Electromagnetically Induced Two Hadron Emission, Lund, Sweden, June 2001 (unpublished).

[61] J. E. Amaro, M. B. Barbaro, J. A. Caballero, T. W. Donnelly, A. Molinari, and I. Sick, Phys. Rev. C 71, 015501 (2005).

[62] J. E. Amaro, M. B. Barbaro, J. A. Caballero, and T. W. Donnelly, nucl-th/0612046.

[63] W. M. Alberico, T. W. Donnelly, and A. Molinari, Nucl. Phys. A512, 541 (1990).

[64] D. Pines, K. F. Quader, and J. Wambach, Nucl. Phys. A477, 365 (1988).

[65] C. Mahaux and H. Ngô, Phys. Lett. B100, 285 (1981).

[66] Y. Horikawa, F. Lenz, and N. C. Mukhopadhyay, Phys. Rev. C 22, 1680 (1980).

[67] J. M. Udias et al. (in preparation). 